\title{
Flows of a Rarefied Gas between Coaxial Circular Cylinders with Nonuniform Surface Properties
}

\author{
Toshiyuki Doi \\ Department of Applied Mathematics and Physics, Graduate School of Engineering, Tottori University, Tottori, Japan \\ Email: doi@tottori-u.ac.jp
}

How to cite this paper: Doi, T. (2019) Flows of a Rarefied Gas between Coaxial Circular Cylinders with Nonuniform Surface Properties. Open Journal of Fluid Dynamics, 9, 22-48. https://doi.org/10.4236/ojfd.2019.91002

Received: December 12, 2018

Accepted: February 12, 2019

Published: February 15, 2019

Copyright $\odot 2019$ by author(s) and Scientific Research Publishing Inc. This work is licensed under the Creative Commons Attribution International License (CC BY 4.0).

http://creativecommons.org/licenses/by/4.0/

(c) () Open Access

\begin{abstract}
Flows of a rarefied gas between coaxial circular cylinders with nonuniform surface properties are studied on the basis of kinetic theory. It is assumed that the outer cylinder is a diffuse reflection boundary and the inner cylinder is a Maxwell-type boundary whose accommodation coefficient varies in the circumferential direction. Three fundamental flows are studied: 1) a flow caused by the rotation of the outer cylinder (Couette flow), 2) a flow induced between the cylinders at rest kept at different temperatures (heat transfer problem), and 3) a flow induced by the circumferential temperature distribution along the cylindrical surfaces (thermal creep flow). The linearized ES-BGK model of the Boltzmann equation is numerically analyzed using a finite difference method. The time-independent behavior of the gas is studied over a wide range of the gas rarefaction degree, the radii ratio, and a parameter characterizing the distribution of the accommodation coefficient. Due to an effect of nonuniform surface properties, a local heat transfer occurs between the gas and the cylindrical surfaces in Couette flow; a local tangential stress arises in the heat transfer problem. However, the total heat transfer between the two cylinders in Couette flow and the total torque acting on the inner cylinder in the heat transfer problem vanish irrespective of the flow parameters. Two nondegenerate reciprocity relations arise due to the effect of nonuniform surface properties. The reciprocity relations among the above-mentioned three flows are numerically confirmed over a wide range of the flow parameters. The force on the inner cylinder, which also arises due to the effect of nonuniform surface properties in Couette flow and the heat transfer problems, is studied.
\end{abstract}

\section{Keywords}

Rarefied Gas Flow, Micro Flow, Boltzmann Equation, Gas-Surface 
Interaction, Reciprocity Relations

\section{Introduction}

Internal flows of a rarefied gas have wide applications, e.g., in vacuum science and micro engineering, and have been studied extensively on the basis of kinetic theory (see Refs. [1]-[7] and the references therein). One of the characteristics of a rarefied gas flow is that the transports of mass and energy are strongly coupled due to the nonequilibrium effect. For example, in a flow between plane parallel walls caused by a pressure gradient (Poiseuille flow), or in a flow caused by a tangential motion of the wall (Couette flow), heat flows are induced in the gas simultaneously with the motion of the gas. These heat flows are, however, parallel to the wall surfaces, and do not contribute to a heat transfer between the gas and the wall surfaces.

On the other hand, another characteristic of a rarefied gas flow is that it is affected by the gas-surface interaction law (surface properties, in short). Therefore, a nonuniformness of the surface properties can cause special phenomena. Examples of the flows between plane parallel walls with unequal surface properties are studied, e.g., in Refs. [8]-[13]. Flows between parallel walls with nonuniform surface properties along the wall surface are studied, e.g., in Refs. [14] [15] [16] [17] [18]. In Refs. [15] [17], it is assumed that one wall is a diffuse reflection boundary and the other wall is a Maxwell-type boundary whose accommodation coefficient varies periodically in the flow direction. The following four flows are studied: a flow induced by a temperature distribution along the wall surfaces (thermal creep flow), a flow induced between the walls at rest kept at different temperatures (heat transfer problem), and the above-mentioned Poiseuille and Couette flows. In this setting, the flow is no longer parallel, and thus, a heat transfer between the gas and the wall surfaces occurs due to the combined effects of gas rarefaction and nonuniform surface properties. In fact, a tangential motion of the wall causes a local heat transfer on the wall surface in Couette flow. In the heat transfer problem, on the other hand, a temperature difference between the walls induces a gas motion and a tangential stress on the wall surface. One of the interesting results in Ref. [17] is that the integrals of these heat flux and the tangential stress over one period of the variation of the accommodation coefficient vanish irrespective of the gas rarefaction degree and the distribution of the accommodation coefficient. To the best of the author's knowledge, this vanishing property has not yet been derived from the Boltzmann equation and the theory of reciprocity [19] [20]. It is possible that this property may have some connection with "the nonexistence of one-way flow" in a pipe or channel by a periodic temperature distribution in rarefied gas dynamics [7]. Naturally, a question arises whether this vanishing property is a special characteristic limited to the plane channel flow or it has a universality over a wider class of flow prob- 
lems.

In this paper, we study slow rarefied gas flows between coaxial circular cylinders with nonuniform surface properties on the basis of kinetic theory. In accordance with Refs. [15] [17], we assume that the outer cylinder is a diffuse reflection boundary and the inner cylinder is a Maxwell-type boundary whose accommodation coefficient varies in the circumferential direction. The following three flows are studied: 1) a flow caused by the rotation of the outer cylinder (Couette flow), 2) a flow induced between the cylinders at rest kept at different temperatures (heat transfer problem), and 3) a flow induced by a circumferential temperature distribution along the cylindrical surfaces at rest (thermal creep flow). The linearized ES-BGK model [21] [22] of the Boltzmann equation is numerically analyzed using a finite difference method. The time-independent behavior of the gas is studied over a wide range of the gas rarefaction degree, the radii ratio, and a parameter characterizing the distribution of the accommodation coefficient. Special attention is focused on the heat flux and the tangential stress on the cylindrical surfaces that arise due to the effect of nonuniform surface properties, and on the resultant heat transfer between and the torque acting on the cylinders. The goals of this paper are as follows. First, it is clarified whether or not the net heat transfer in Couette flow and the torque in the heat transfer problem vanish irrespective of the flow parameters. Second, three reciprocity relations among the above-mentioned three flows are numerically confirmed over a wide range of the flow parameters. Note that in a conventional Couette flow and heat transfer problems in which the accommodation coefficient is uniform, it is obvious that the flow is axially symmetric and the cylinders are subject to no force. The third goal is therefore to study the force acting on the inner cylinder arising from the effect of nonuniform surface properties. To the best of the author's knowledge, studies on relations among several flows between boundaries with tangentially nonuniform surface properties are not available except in Refs. [17] [18] and in the present paper. In this paper, we use the simplest model, i.e., the Maxwell-type boundary condition, to model the nonuniform surface property. For, this model is characterized by only one parameter (accommodation coefficient), and thus convenient to study over various distributions of the surface property and other parameters. Incidentally, the flow problems considered in this paper are simple models of micro lubrication and heat transfer between a journal and a bearing in which the journal is partially soiled or fabricated with various materials. Studies on these flows are therefore technically valuable in designing a micro lubrication and a heat transfer in micro-electro-mechanical systems (MEMS).

This paper is organized as follows. The problem, basic equations, and basic properties of the solutions are described in Section 2. The numerical method, computational condition, and the results of accuracy tests are summarized in Section 3. The results and the discussion are presented in Section 4. Finally, a conclusion is given in Section 5 . 


\section{Problem and Basic Equation}

\subsection{Problem}

Let us consider a rarefied gas between the coaxial circular cylinders placed at $X_{1}^{2}+X_{2}^{2}=r_{0}^{2}$ and $X_{1}^{2}+X_{2}^{2}=r_{1}^{2}$, where $\left(X_{1}, X_{2}, X_{3}\right)$ is a Cartesian coordinate system and $r_{0}$ and $r_{1}$ are constants such that $0<r_{0}<r_{1}$ (Figure $1(a))$. The outer cylinder is a diffuse reflection boundary, whose temperature is denoted by $T_{w 1}$. The inner cylinder is a Maxwell-type boundary at rest whose accommodation coefficient $\alpha$ varies in the circumferential direction. To be specific, by introducing the cylindrical coordinates $(r, \theta, z)$ defined by

$$
X_{1}=r \cos \theta, \quad X_{2}=r \sin \theta, \quad X_{3}=z \quad(0 \leq \theta<2 \pi),
$$

$\alpha$ is the function of the azimuthal angle $\theta$ :

$$
\alpha(\theta)=\left\{\begin{array}{l}
\frac{\alpha_{2}+\alpha_{1}}{2}+\frac{\alpha_{2}-\alpha_{1}}{2} \cos \frac{\theta}{2 C} \quad(0 \leq \theta \leq 2 \pi C), \\
\frac{\alpha_{2}+\alpha_{1}}{2}+\frac{\alpha_{2}-\alpha_{1}}{2} \cos \frac{2 \pi-\theta}{2(1-C)} \quad(2 \pi C<\theta<2 \pi) .
\end{array}\right.
$$

Here, $\alpha_{1}, \alpha_{2}$, and $C$ are constants such that $0 \leq \alpha_{1} \leq 1, \quad 0 \leq \alpha_{2} \leq 1,0<C<1$ (see Figure 1(b)). The temperature of this wall is denoted by $T_{w 0}$. We study the following three flows: 1) a flow caused by the rotation of the outer cylinder (Couette flow), where the angular velocity is $\Omega$ and the cylinders are kept at the uniform temperature $\left.T_{w 0}=T_{w 1}=T_{0} ; 2\right)$ a flow induced between the cylinders at rest kept at different temperatures $T_{w 0}=T_{0}$ and $T_{w 1}=T_{0}\left(1+\Delta_{\mathrm{HT}}\right)$ (heat transfer problem); and 3) a flow induced by the temperature distribution $T_{w 0}=T_{w 1}=T_{0}\left(1+\Delta_{\mathrm{TC}} X_{1} / r_{0}\right)$ along the cylindrical surfaces at rest, where $T_{0}, \Delta_{\mathrm{HT}}$, and $\Delta_{\mathrm{TC}}$ are constants. The time-independent behavior of the gas is studied on the basis of kinetic theory.

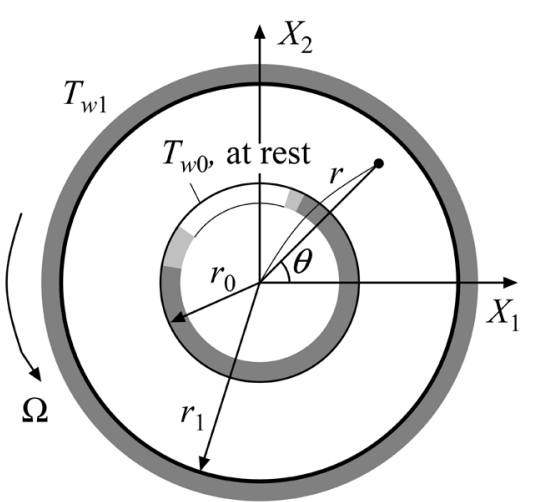

(a)

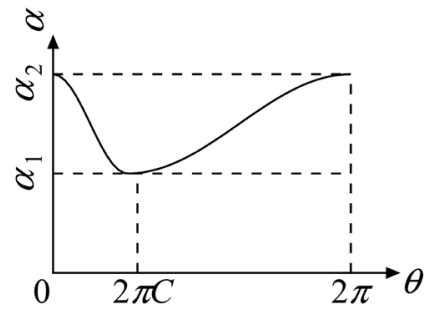

(b)

Figure 1. Schematic view of the system. (a) Schematic view from the $X_{3}$ direction and (b) distribution of the accommodation coefficient $\alpha(\theta)$ along the surface of the inner cylinder (Equation (2)). In panel (a), the white part of the surface of the inner cylinder represents the interval in which $\alpha$ is close to the minimum value $\alpha_{1}$. In panel (b), the distribution $\alpha(\theta)$ is plotted for $C=0.25$. 
In this paper, we assume that the gas behavior is governed by the ES-BGK model of the Boltzmann equation with the Prandtl number 2/3. It is also assumed that the causes of the flows are so small that the equation and the boundary condition may be linearized. To be specific, we assume that $r_{1}|\Omega| / V_{0} \ll 1$, $\left|\Delta_{\mathrm{HT}}\right| \ll 1$, and $\left|r_{1} r_{0}^{-1} \Delta_{\mathrm{TC}}\right| \ll 1$, where $V_{0}=\left(2 k_{B} T_{0} / m\right)^{1 / 2}, k_{B}$ is the Boltzmann constant, and $m$ is the mass of a molecule.

\subsection{Basic Equation}

Let us take $r_{0}, V_{0}$, and $\rho_{0}$ as the reference length, the reference speed, and the reference density of the system, where $\rho_{0}$ is the mean density of the gas over the annulus domain $r_{0}<r<r_{1}, 0<z<r_{0}$. We use the dimensionless variables $\hat{r}$ and $\hat{z}$ for the spatial coordinates $r$ and $z$, and $\zeta_{l}(l=r, \theta, z)$ for the molecular velocity components $\xi_{l}$ [7]:

$$
\hat{r}=\frac{r}{r_{0}}, \quad \hat{z}=\frac{z}{r_{0}}, \quad \zeta_{l}=\frac{\xi_{l}}{V_{0}} .
$$

The dimensionless molecular velocity is also denoted by $\zeta$. The perturbation $\phi$ of the velocity distribution function $f$ from the equilibrium state at rest with the density $\rho_{0}$ and the temperature $T_{0}$ is defined by

$$
\phi=\frac{f}{\rho_{0} V_{0}^{-3} E}-1,
$$

where $E(\zeta)=\pi^{-3 / 2} \exp \left(-\zeta^{2}\right)$ with $\zeta^{2}=\zeta_{r}^{2}+\zeta_{\theta}^{2}+\zeta_{z}^{2}$. Correspondingly, the perturbations of the macroscopic variables $\omega, u_{t}, \tau, P, P_{t \gamma}, Q_{t}$

$(l=r, \theta, z ; \gamma=r, \theta, z)$ for the density $\rho$, the flow velocity $v_{l}$, the temperature $T$, the pressure $p$, the stress tensor $p_{t \gamma}$, and the heat flow vector $q_{l}$ are defined by

$$
\begin{array}{ll}
\omega=\rho / \rho_{0}-1, & u_{t}=v_{\iota} / V_{0}, \quad \tau=T / T_{0}-1, \\
P=p / p_{0}-1, & P_{\imath \gamma}=p_{\imath \gamma} / p_{0}-\delta_{t \gamma}, \quad Q_{\imath}=q_{\imath} /\left(p_{0} V_{0}\right),
\end{array}
$$

where $p_{0}=k_{B} \rho_{0} T_{0} / m$.

The linearized ES-BGK model of the Boltzmann equation with the Prandtl number $2 / 3$ in the time-independent and axially uniform $(\partial \phi / \partial \hat{z}=0)$ state is written in the cylindrical coordinate system as

$$
\begin{gathered}
\zeta_{r} \frac{\partial \phi}{\partial \hat{r}}+\frac{\zeta_{\theta}}{\hat{r}} \frac{\partial \phi}{\partial \theta}+\frac{\zeta_{\theta}^{2}}{\hat{r}} \frac{\partial \phi}{\partial \zeta_{r}}-\frac{\zeta_{\theta} \zeta_{r}}{\hat{r}} \frac{\partial \phi}{\partial \zeta_{\theta}} \\
=\frac{2}{\sqrt{\pi} K n}\left[-\phi+\omega+2 \zeta_{r} u_{r}+2 \zeta_{\theta} u_{\theta}+\left(\zeta^{2}-\frac{3}{2}\right) \tau\right. \\
\left.-\frac{1}{2}\left(\zeta_{r}^{2} P_{r r}+\zeta_{\theta}^{2} P_{\theta \theta}+\zeta_{z}^{2} P_{z z}+2 \zeta_{\theta} \zeta_{r} P_{\theta r}-\zeta^{2} P\right)\right], \\
\omega=\int \phi E \mathrm{~d} \zeta, \\
u_{t}=\int \zeta_{l} \phi E \mathrm{~d} \zeta, \\
\tau=\frac{2}{3} \int\left(\zeta^{2}-\frac{3}{2}\right) \phi E \mathrm{~d} \zeta,
\end{gathered}
$$




$$
\begin{gathered}
P=\omega+\tau, \\
P_{l \gamma}=2 \int \zeta_{l} \zeta_{\gamma} \phi E \mathrm{~d} \zeta, \\
Q_{t}=\int \zeta_{t}\left(\zeta^{2}-\frac{5}{2}\right) \phi E \mathrm{~d} \zeta .
\end{gathered}
$$

In (6), $K n=\ell / r_{0}$ is the Knudsen number; $\ell$ is the mean free path of the gas in the equilibrium state at rest with the density $\rho_{0}$ and the temperature $T_{0}$. The mean free path $\ell$ is related to the viscosity $\mu$ and the heat conductivity $\lambda$ of the gas as [23]

$$
\mu=\frac{\sqrt{\pi}}{3} \frac{p_{0}}{V_{0}} \ell, \quad \lambda=\frac{5 \sqrt{\pi}}{4} \frac{k_{B} p_{0}}{m V_{0}} \ell .
$$

In (7)-(12) and in what follows, $\mathrm{d} \zeta=\mathrm{d} \zeta_{r} \mathrm{~d} \zeta_{\theta} \mathrm{d} \zeta_{z}$, and the range of integration with respect to $\zeta_{l}$ is its whole space unless otherwise stated.

The boundary conditions on the cylindrical walls are

$$
\begin{aligned}
& \phi\left(\zeta_{r}\right)=(1-\alpha) \phi\left(-\zeta_{r}\right)+\alpha\left[\left(\zeta^{2}-2\right) \tau_{w 0}-2 \sqrt{\pi} \int_{\zeta_{r^{*}<0}} \zeta_{r^{*}} \phi\left(\zeta_{*}\right) E\left(\zeta_{*}\right) \mathrm{d} \zeta_{*}\right] \\
& \left(\hat{r}=1, \zeta_{r}>0\right), \\
& \phi=2 \zeta_{\theta} u_{w}+\left(\zeta^{2}-2\right) \tau_{w 1}+2 \sqrt{\pi} \int_{\zeta_{r^{*}>0}} \zeta_{r^{*}} \phi\left(\zeta_{*}\right) E\left(\zeta_{*}\right) \mathrm{d} \zeta_{*} \quad\left(\hat{r}=R, \zeta_{r}<0\right),
\end{aligned}
$$

where $\alpha=\alpha(\theta), R=r_{1} / r_{0}$ is the radii ratio, and $\phi\left( \pm \zeta_{r}\right)$ denotes the abbreviation for $\phi\left(\hat{r}, \theta, \pm \zeta_{r}, \zeta_{\theta}, \zeta_{z}\right)$. The $\tau_{w 0}, \tau_{w 1}$, and $u_{w}$ are defined by

$$
\begin{aligned}
& \tau_{w 0}=\tau_{w 1}=0, u_{w}=R \Delta_{\mathrm{CF}} \quad(\text { Couette flow }), \\
& \tau_{w 0}=0, \tau_{w 1}=\Delta_{\mathrm{HT}}, u_{w}=0 \quad(\text { heat transfer problem), } \\
& \tau_{w 0}=\tau_{w 1} / R=\Delta_{\mathrm{TC}} \cos \theta, u_{w}=0 \quad \text { (thermal creep flow), }
\end{aligned}
$$

where $\Delta_{\mathrm{CF}}=r_{0} \Omega / V_{0}$. The periodic condition at the cross section $\theta=2 \pi$ is

$$
\begin{array}{cc}
\phi\left(\hat{r}, 0, \zeta_{r}, \zeta_{\theta}, \zeta_{z}\right)=\phi\left(\hat{r}, 2 \pi, \zeta_{r}, \zeta_{\theta}, \zeta_{z}\right) & \left(\zeta_{\theta}>0\right), \\
\phi\left(\hat{r}, 2 \pi, \zeta_{r}, \zeta_{\theta}, \zeta_{z}\right)=\phi\left(\hat{r}, 0, \zeta_{r}, \zeta_{\theta}, \zeta_{z}\right) & \left(\zeta_{\theta}<0\right) .
\end{array}
$$

Finally, from the definition of the average density $\rho_{0}$, the solution should satisfy

$$
\int_{0}^{2 \pi} \mathrm{d} \theta \int_{1}^{R} \mathrm{~d} \hat{r} \hat{r} \int \phi E \mathrm{~d} \zeta=0
$$

From (6)-(18), it is easily seen that we can seek the solution $\phi$ that is symmetric with respect to $\zeta_{z}$.

From now on, we denote the solution for Couette flow, heat transfer, and the thermal creep problems, respectively, by the subscript CF, HT, and TC, e.g., $\phi_{\mathrm{CF}}$, if necessary. The subscript is frequently represented by the dummy letter J, e.g., $\phi_{\mathrm{J}}$ with $\mathrm{J}=\mathrm{CF}, \mathrm{HT}$, and TC.

The boundary value problem (6)-(18) is characterized by the following dimensionless parameters

$$
K n=\frac{\ell}{r_{0}}, \quad R=\frac{r_{1}}{r_{0}}, \quad \alpha_{1}, \alpha_{2} \text { and } C .
$$


We will solve the problem over a wide range of the parameters (19). Among the parameters (19), $\alpha_{1}, \alpha_{2}$, and $C$ are those characterizing the distribution of the accommodation coefficient $\alpha(\theta)$ in (2). The last parameter $C$ represents a degree of asymmetry of the distribution $\alpha(\theta)$ with respect to $\theta=\pi$; the $\alpha(\theta)$ is symmetric when $C=1 / 2$ and the degree of asymmetry increases as $|1 / 2-C|$ increases.

Multiplying (6) by $E, 2 \zeta_{\theta} E$, and $\zeta^{2} E$, and integrating with respect to $\zeta$, we obtain the conservation of mass, momentum, and energy:

$$
\begin{gathered}
\frac{1}{\hat{r}} \frac{\partial \hat{r} u_{r}}{\partial \hat{r}}+\frac{1}{\hat{r}} \frac{\partial u_{\theta}}{\partial \theta}=0, \\
\frac{1}{\hat{r}^{2}} \frac{\partial \hat{r}^{2} P_{\theta r}}{\partial \hat{r}}+\frac{1}{\hat{r}} \frac{\partial P_{\theta \theta}}{\partial \theta}=0, \\
\frac{1}{\hat{r}} \frac{\partial \hat{r} Q_{r}}{\partial \hat{r}}+\frac{1}{\hat{r}} \frac{\partial Q_{\theta}}{\partial \theta}=0 .
\end{gathered}
$$

Integrating (20)-(22) over $0<\theta<2 \pi$ and applying the boundary condition (13) or (14), we obtain

$$
\int_{0}^{2 \pi} u_{r} \mathrm{~d} \theta=0, \quad \hat{r}^{2} \int_{0}^{2 \pi} P_{\theta r} \mathrm{~d} \theta=c_{1}, \quad \hat{r} \int_{0}^{2 \pi} Q_{r} \mathrm{~d} \theta=c_{2},
$$

where $c_{1}$ and $c_{2}$ are constants. The second relation of (23) is the conservation law of angular momentum.

\subsection{Force, Torque, Heat Flow Rate, and the Reciprocity Relations}

Once the macroscopic variables, e.g. $P_{\theta r}(\hat{r}, \theta)$, are obtained, the force, torque, and the heat flow rate on the cylinders are obtained by the integral of the macroscopic variables as follows. The Cartesian components $\left(F_{1}, F_{2}, 0\right)$ of the force acting on the inner cylinder per unit length in the axial direction are given by

$$
\frac{1}{p_{0} r_{0}}\left(\begin{array}{l}
F_{1} \\
F_{2}
\end{array}\right)=-\int_{0}^{2 \pi}\left(\begin{array}{l}
P_{r r} \cos \theta-P_{\theta r} \sin \theta \\
P_{r r} \sin \theta+P_{\theta r} \cos \theta
\end{array}\right) \mathrm{d} \theta \quad(\hat{r}=1)
$$

For the convenience of the following discussion, we define the normalized torque $\mathcal{T}_{\mathrm{J}}(\hat{r})$ acting on and the heat flow rate $\mathcal{Q}_{\mathrm{J}}(\hat{r})$ onto the surface $\hat{r}=\hat{r}, 0 \leq \theta<2 \pi$ from the outer per unit length in the $\hat{z}$ direction:

$$
\begin{aligned}
& \mathcal{T}_{\mathrm{J}}(\hat{r})=-\frac{1}{\Delta_{\mathrm{J}}} \int_{0}^{2 \pi} \hat{r}^{2} P_{\theta r \mathrm{~J}}(\hat{r}, \theta) \mathrm{d} \theta, \\
& \mathcal{Q}_{\mathrm{J}}(\hat{r})=-\frac{1}{\Delta_{\mathrm{J}}} \int_{0}^{2 \pi} \hat{r} Q_{r \mathrm{~J}}(\hat{r}, \theta) \mathrm{d} \theta,
\end{aligned}
$$

where $\mathrm{J}=\mathrm{CF}, \mathrm{HT}$, and TC. The $\mathcal{T}_{\mathrm{J}}$ and $\mathcal{Q}_{\mathrm{J}}$ are independent of $\hat{r}$ due to the conservation laws (23), so that the position $\hat{r}$ of evaluation may be omitted. The dimensional torque and the heat flow rate are, respectively, given by $p_{0} r_{0}^{2} \Delta_{\mathrm{J}} \mathcal{T}_{\mathrm{J}}$ and $p_{0} r_{0} V_{0} \Delta_{\mathrm{J}} \mathcal{Q}_{\mathrm{J}}$.

From the symmetric relation of the linearized Boltzmann equation [2] [19] 
[20], the following three reciprocity relations hold among the three flow problems $(\mathrm{J}=\mathrm{CF}, \mathrm{HT}$, and TC) for arbitrary values of the parameters (19):

$$
\begin{gathered}
\mathcal{Q}_{\mathrm{CF}}(R)=-\mathcal{T}_{\mathrm{HT}}(R), \\
\mathcal{T}_{\mathrm{TC}}(R)=\mathcal{H}_{\mathrm{CF}}, \\
\mathcal{Q}_{\mathrm{TC}}(R)=-\mathcal{H}_{\mathrm{HT}},
\end{gathered}
$$

where

$$
\mathcal{H}_{\mathrm{J}}=\frac{1}{\Delta_{\mathrm{J}}} \int_{0}^{2 \pi}\left[R^{2} Q_{r \mathrm{~J}}(R, \theta)-Q_{r \mathrm{~J}}(1, \theta)\right] \cos \theta \mathrm{d} \theta .
$$

Equations (27)-(29) will be numerically confirmed over a wide range of the parameters (19).

\subsection{Cases of Uniform or Symmetric Distribution of the Accommodation Coefficient}

When the accommodation coefficient $\alpha(\theta)$ in the boundary condition (13) is uniform (conventional case, in short), the solutions of the boundary value problem (6)-(18) are simplified as follows. The solutions for the conventional cases are reported, e.g., in Refs. [24] [25] for Couette flow and Ref. [26] for the heat transfer problem.

Couette flow and the heat transfer problems reduce to axially symmetric problems, i.e., $\partial \phi / \partial \theta=0$, in the conventional case. In Couette flow problem, further, it is easily seen that the distribution function $\phi$ is an odd function with respect to $\zeta_{\theta}$. Consequently from (7)-(12), we directly obtain

$$
\omega_{\mathrm{CF}}=u_{r \mathrm{CF}}=\tau_{\mathrm{CF}}=P_{r r \mathrm{CF}}=P_{\theta \theta \mathrm{CF}}=P_{z z \mathrm{CF}}=Q_{r \mathrm{CF}}=0 \text {, }
$$

and thus

$$
F_{1 \mathrm{CF}}=F_{2 \mathrm{CF}}=\mathcal{Q}_{\mathrm{CF}}=\mathcal{H}_{\mathrm{CF}}=0 .
$$

In the heat transfer problem, similarly, the distribution function $\phi$ is an even function with respect to $\zeta_{\theta}$. By using the conservation laws (23) and the boundary condition (13) or (14), we obtain

$$
u_{r \mathrm{HT}}=u_{\theta \mathrm{HT}}=P_{\theta r \mathrm{HT}}=Q_{\theta \mathrm{HT}}=0,
$$

and

$$
F_{1 \mathrm{HT}}=F_{2 \mathrm{HT}}=\mathcal{T}_{\mathrm{HT}}=\mathcal{H}_{\mathrm{HT}}=0 .
$$

In the thermal creep flow problem, in contrast, the boundary value problem remains a spatially two dimensional one for $\hat{r}$ and $\theta$. Because of the special form of the temperature distribution $\tau_{w 0}$ and $\tau_{w 1}$ in (15), however, we find that the solution $\phi=\phi_{e} \cos \theta+\phi_{o} \sin \theta$ is compatible with the boundary value problem (6)-(18), where $\phi_{e}$ and $\phi_{o}$ are independent of $\theta$ and, respectively, even and odd functions with respect to $\zeta_{\theta}$. From this form, we see that $P_{\theta r \mathrm{TC}} / \sin \theta, P_{r r \mathrm{TC}} / \cos \theta$, and $Q_{r \mathrm{TC}} / \cos \theta$ are independent of $\theta$, and thus

$$
F_{2 \mathrm{TC}}=\mathcal{T}_{\mathrm{TC}}=\mathcal{Q}_{\mathrm{TC}}=0 .
$$


To summarize, when the accommodation coefficient is uniform, both sides of all the reciprocity relations (27)-(29) vanish; obviously, the cylinders are subject to no force in Couette and heat transfer problems. Probably because of this reason, the reciprocity relations are rarely discussed in the flow problems between coaxial circular cylinders. In the case of nonuniform accommodation coefficient to be studied in this paper, in contrast, (32), (34), and (35) do not generally hold, and all the three reciprocity relations (27)-(29) are of interest. The numerical confirmation of the reciprocity relations (27)-(29) is one of the important goals of this paper.

Special attention is focused on the quantities in (27). In Refs. [15] [17], flow problems between plane parallel walls in which one of the walls has a periodic distribution of the accommodation coefficient were studied. In Ref. [17], due to the effect of the nonuniform accommodation coefficient of the solid boundary, a local heat transfer across the wall surface occurs in plane Couette flow, and a local tangential stress on the surface occurs in the plane heat transfer problem. The numerical solution in Ref. [17] showed, however, that the integrals of the heat flux and the tangential stress over one period of the distribution of the accommodation coefficient vanish irrespective of the flow parameters (see Section 1). If this phenomenon has a universality beyond the flows between plane parallel walls, both sides of (27) may vanish. To clarify this point is an important motivation of this study.

Let us discuss another special case that the accommodation coefficient $\alpha(\theta)$ is nonuniform but symmetric with respect to $\theta=\pi$ (symmetric case, in short). This is the case of $C=1 / 2$ in (2). In this case, all the reciprocity relations (27)-(29) degenerate as follows. The derivation is quite similar to that in Ref. [15] and thus omitted. In Couette flow problem, in this case, the macroscopic variables $u_{\theta \mathrm{CF}}, P_{\theta r \mathrm{CF}}$, and $Q_{\theta \mathrm{CF}}$ are symmetric with respect to $\theta=\pi$, whereas $\omega_{\mathrm{CF}}, u_{r \mathrm{CF}}, \tau_{\mathrm{CF}}, P_{r r \mathrm{CF}}$, and $Q_{r \mathrm{CF}}$ are antisymmetric. Therefore,

$$
F_{1 \mathrm{CF}}=\mathcal{Q}_{\mathrm{CF}}=\mathcal{H}_{\mathrm{CF}}=0 \text {. }
$$

In the heat transfer and the thermal creep problems, $\omega_{\mathrm{J}}, u_{r \mathrm{~J}}, \tau_{\mathrm{J}}, P_{r \mathrm{r} \mathrm{J}}$, and $Q_{r \mathrm{~J}}$ (J $=\mathrm{HT}$ and TC) are symmetric with respect to $\theta=\pi$, whereas $u_{\theta \mathrm{J}}, P_{\theta r \mathrm{~J}}$, and $Q_{\theta \mathrm{J}}$ are antisymmetric. Therefore,

$$
F_{2 \mathrm{~J}}=\mathcal{T}_{\mathrm{J}}=0 \quad(\mathrm{~J}=\mathrm{HT} \text { and } \mathrm{TC}) .
$$

That is, all the reciprocity relations (27)-(29) degenerate also in the symmetric case. In this paper, therefore, the cases other than $C=1 / 2$ are included.

\section{Numerical Analysis}

\subsection{Numerical Method}

A few preliminary processes are explained. First, the new variables $\zeta_{\rho}$ and $\theta_{\zeta}$ are introduced in place of $\zeta_{r}$ and $\zeta_{\theta}$ [7]:

$$
\zeta_{r}=\zeta_{\rho} \cos \theta_{\zeta}, \quad \zeta_{\theta}=\zeta_{\rho} \sin \theta_{\zeta} \quad\left(\zeta_{\rho}>0,-\pi<\theta_{\zeta} \leq \pi\right)
$$


Then, the number of derivative terms in (6) is reduced from four to three, which is convenient for the numerical analysis. Second, by introducing the marginal distribution functions $\Phi$ and $\Theta$ defined by [7]

$$
\Phi=\frac{1}{\sqrt{\pi}} \int_{-\infty}^{\infty} \phi \exp \left(-\zeta_{z}^{2}\right) \mathrm{d} \zeta_{z}, \quad \Theta=\frac{2}{\sqrt{\pi}} \int_{-\infty}^{\infty} \zeta_{z}^{2} \phi \exp \left(-\zeta_{z}^{2}\right) \mathrm{d} \zeta_{z},
$$

the molecular velocity component $\zeta_{z}$ can be eliminated from the boundary value problem (6)-(18). Then, we are led to a boundary value problem for the four independent variables $\hat{r}, \theta, \zeta_{\rho}$, and $\theta_{\zeta}$.

In the numerical analysis, the infinite interval $\zeta_{\rho}>0$ is replaced by a finite one $0<\zeta_{\rho} \leq \zeta_{D}$, where $\zeta_{D}$ is a constant. The computational grid system is arranged in the four-dimensional space $1 \leq \hat{r} \leq R, 0 \leq \theta \leq 2 \pi, 0<\zeta_{\rho} \leq \zeta_{D}$, and $-\pi \leq \theta_{\zeta} \leq \pi$. Then, a finite difference scheme of the second order with respect to $\hat{r}, \theta$, and $\theta_{\zeta}$ is constructed. The time-independent solution is obtained by an iteration method.

A few remarks on the numerical method are given below. First, the solution $\phi$ of the boundary-value problem is discontinuous across the surfaces

$$
\hat{r} \sin \theta_{\zeta}= \pm 1, \quad\left|\theta_{\zeta}\right| \leq \pi / 2, \quad 0 \leq \theta \leq 2 \pi,
$$

in the $\left(\hat{r}, \theta, \theta_{\zeta}\right)$ space. Therefore, a conventional finite difference scheme cannot be applied directly. In this study, the hybrid scheme of finite difference and characteristic coordinate methods devised in Refs. [27] [28] is adopted. Incidentally, if the outer cylinder is the Maxwell-type boundary as well, the discontinuity staring from the inner cylinder reflects on the outer cylinder, and the discontinuous surfaces are present also in the region of $\left|\theta_{\zeta}\right|>\pi / 2$. In this case, the construction of the numerical scheme is more difficult. On the other hand, if the inner cylinder rotates, the problem is obviously a time-dependent one and elaborate to analyze. This is the reason why we chose the situation that the outer cylinder is a diffuse reflection boundary and the inner cylinder is at rest in this paper. Second, it is difficult to obtain an accurate solution at small Knudsen numbers because of the numerical error arising from the collision term [5]. To avoid this difficulty, we subtract the asymptotic solution [5] [23] for small Knudsen numbers from the numerical solution in calculating the collision term, or (7)-(12). In this paper, the asymptotic solution is constructed approximately using the numerical values of the macroscopic variables. This subtraction process is adopted when $K n /(R-1) \leq 1$.

\subsection{Computational Condition}

The computational grid points are arranged as follows. For the coordinate $\hat{r}$, $N_{r}$ grid points are arranged in the interval $1 \leq \hat{r} \leq R$, where grid size is uniform $\left(=d_{r 2}(R-1)\right)$ in $|(\hat{r}-1) /(R-1)-1 / 2|<0.34$ and nonuniform otherwise; the grid size is minimum $\left(=d_{r 1}(R-1)\right)$ at $\hat{r}=1$ and $R$. For the coordinate $\theta$, 101 grid points are arranged uniformly in the interval $0 \leq \theta \leq 2 \pi$. For the coordinate $\zeta_{\rho}, 31$ grid points are arranged uniformly in $0 \leq \zeta_{\rho} \leq \zeta_{D}$, where we 
chose $\zeta_{D}=5$. For the coordinate $\theta_{\zeta}, N_{\theta}$ grid points are arranged uniformly in $-\pi \leq \theta_{\zeta} \leq \pi$. Here, $N_{r}, N_{\theta}, d_{r 1}$, and $d_{r 2}$ are constants chosen depending on the parameters (19) as follows:

$\left(N_{r}, N_{\theta}, d_{r 1}, d_{r 2}\right)=\left(1281,401,2.7 \times 10^{-5}, 1.3 \times 10^{-3}\right)$ when $K n \leq 0.2$ and $R=5$, or $\left(N_{r}, N_{\theta}, d_{r 1}, d_{r 2}\right)=\left(641,641,5.4 \times 10^{-5}, 2.5 \times 10^{-3}\right)$ otherwise.

\subsection{Results of Accuracy Tests}

The accuracy of the numerical solutions is tested as follows. The following tests are conducted over all the combinations of $R=1.2,2,5, C=0.1,0.25, \alpha_{1}=0.5$, $\alpha_{2}=1$, and $K n=0.1,1,10$ of the parameters (19).

1) Conservation law. The solution of the boundary value problem (6)-(18) should satisfy the conservation laws (23). That is, the left-hand sides in (23) should be independent of $\hat{r}$. Therefore, the maximum variation of the left-hand side in the numerical solution in $1 \leq \hat{r} \leq R$ serves to estimate the numerical error. The maximum variations of the left-hand side of the second relation of (23) in Couette and thermal creep problems are, respectively, less than $0.63 \%$ and $0.45 \%$ relative to the value at $\hat{r}=1$. The maximum variations of the left-hand side of the third relation in heat transfer and thermal creep problems are, respectively, less than $0.072 \%$ and $2.4 \%$ relative to its value at $\hat{r}=1$. As we will see in Section 4, the left-hand side of the second relation in heat transfer problem and that of the third relation in Couette flow vanish $\left(c_{1}=c_{2}=0\right)$, so that the relative error cannot be defined. Instead, the maximum variations of these two quantities relative to the maximum of the respective integrands $\hat{r}^{2}\left|P_{\theta r \mathrm{HT}}\right|$ and $\hat{r}\left|Q_{r \mathrm{CF}}\right|$ over the cylindrical surfaces are, respectively, $0.008 \%$ and $0.13 \%$. The first relation of (23) is not used for the error estimate because the integrand $u_{r}$ vanishes identically on the cylindrical surfaces by (13) and (14).

2) Re-computation using a different computational grid system. For a test of accuracy, we also conducted re-computations using a coarser grid system. In the coarser system, the grid sizes in $\hat{r}, \theta$, and $\theta_{\zeta}$ are two times coarser, that in $\zeta_{\rho}$ is approximately 1.4 times coarser, and the upper limit $\zeta_{D}$ of $\zeta_{\rho}$ is reduced to $\zeta_{D}=4.5$, simultaneously. The error of the numerical solution using the grid system of Section 3.2 is estimated from the difference between the two numerical solutions. The estimated numerical error in the values of the normalized torque $\mathcal{T}_{\mathrm{CF}}$ and $\mathcal{T}_{\mathrm{TC}}$ (Equation (25)) at $\hat{r}=1$ is $0.076 \%$ and $0.35 \%$, respectively; the error in the normalized heat flow rate $\mathcal{Q}_{\mathrm{HT}}$ and $\mathcal{Q}_{\mathrm{TC}}$ (Equation (26)) at $\hat{r}=1$ is $0.0072 \%$ and $0.36 \%$, respectively. The other quantities $\mathcal{Q}_{\mathrm{CF}}$ and $\mathcal{T}_{\mathrm{HT}}$ will be shown to vanish in Section 4. The numerical error in the force $\left(F_{1}, F_{2}\right)$ (Equation $\left.(24)\right)$ is estimated to be less than $0.14 \%$, $0.32 \%$, and $0.44 \%$ in Couette flow, heat transfer, and thermal creep problems, respectively.

The reciprocity relations (27)-(29) also serve to estimate the accuracy of the numerical solutions. The result will be presented in Section 4.4. 
It may be noted that the accuracy in the numerical solution, e.g., the value of $\mathcal{Q}_{\mathrm{TC}}$, in this paper is worse than that in our previous paper of the flow between plane parallel walls [17]. This is probably because that the basic Equation (6) is complicated (three derivative terms in place of two in Ref. [17]), and that the substantial Knudsen number $\ell /\left(r_{1}-r_{0}\right)$ based on the gap size $r_{1}-r_{0}$ can be very small when $r_{1} / r_{0}=R$ is large. At small Knudsen numbers, it is difficult to obtain an accurate numerical solution not only because the numerical error arising from the collision term is non-negligible [5], but also because the quantities of interest are very small. For example, the worst case of the energy conservation law $(2.4 \%)$ in the item 1) occurs in the thermal creep flow at $(K n, R, C)=(0.1,5,0.1)$. In this case, the normalized energy flow rate $\mathcal{Q}_{\mathrm{TC}}$ is $2.9 \times 10^{-3}$, which is extremely small. Note that the normalized distribution function $\phi_{\mathrm{TC}} / \Delta_{\mathrm{TC}}$ is of the order of unity whereas the heat flow rate $\mathcal{Q}_{\mathrm{TC}}$ is extremely small due to a drastic cancellation in the successive integrals (12) and (26).

\section{Results and Discussion}

In this paper, the numerical computation is conducted for all the combinations of the parameters (19) as follows: $R=1.2,2,5, C=0.1,0.25,0.5, \alpha_{1}=0.5$, $\alpha_{2}=1$, and 15 Knudsen numbers $K n$ ranging between 0.1 and 10 . The results of the numerical analysis are presented in this section. In the following discussion, we also use the dimensionless Cartesian coordinates $x_{1}=X_{1} / r_{0}=\hat{r} \cos \theta$ and $x_{2}=X_{2} / r_{0}=\hat{r} \sin \theta$, and the vector components

$$
u_{1}=u_{r} \cos \theta-u_{\theta} \sin \theta, \quad u_{2}=u_{r} \sin \theta+u_{\theta} \cos \theta,
$$

and so on. Further, it is assumed that $\Delta_{\mathrm{CF}}, \Delta_{\mathrm{HT}}$, and $\Delta_{\mathrm{TC}}$ in (15) are positive without loss of generality.

\subsection{Couette Flow}

First, some examples of the flow field of Couette flow are presented in Figure 2. The flow velocity and the heat flow for the radii ratio $R=2$ and the Knudsen numbers $K n=0.1,1$, and 10 are presented in Figures 2(a)-(c), respectively; those for $R=5$ and the same $K n$ 's are presented in Figures 2(d)-(f), respectively ( $\alpha_{1}=0.5, \alpha_{2}=1, C=0.25$ ). The arrow represents the normalized flow velocity $\left(u_{1 \mathrm{CF}}, u_{2 \mathrm{CF}}\right) / \Delta_{\mathrm{CF}}$ at its starting point, the magnitude of which is shown by the arrow at the upper right corner of each panel. The dotted line represents the "stream line" of the heat flow vector $\left(Q_{\mathrm{ICF}}, Q_{2 \mathrm{CF}}\right)$, i.e., the isoline of the function $\Psi$ defined by

$$
Q_{\mathrm{ICF}}=\frac{\partial \Psi}{\partial x_{2}}, \quad Q_{2 \mathrm{CF}}=-\frac{\partial \Psi}{\partial x_{1}} .
$$

The accommodation coefficient $\alpha(\theta)$ is minimum at $\theta=\pi / 2$ when $C=0.25$; the white part along the surface of the inner cylinder represents the part in which $\alpha$ is close to the minimum $(0.5 \leq \alpha \leq 0.55)$. Now let us examine 


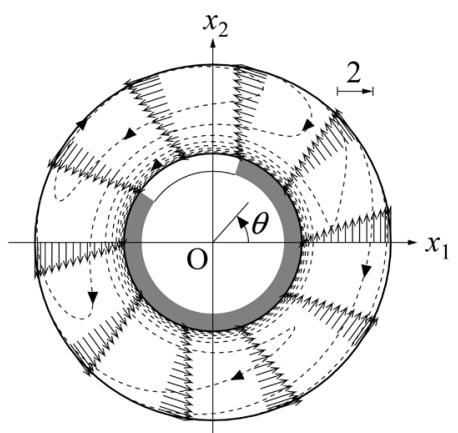

(a) $K n=0.1, R=2$

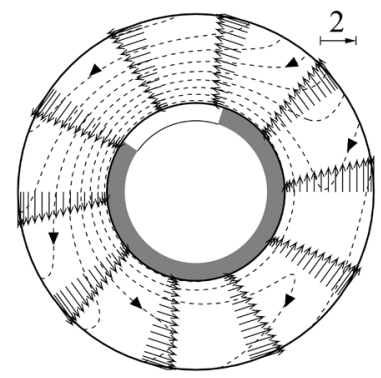

(b) $K n=1, R=2$

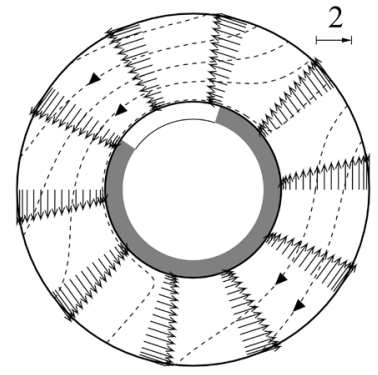

(c) $K n=10, R=2$

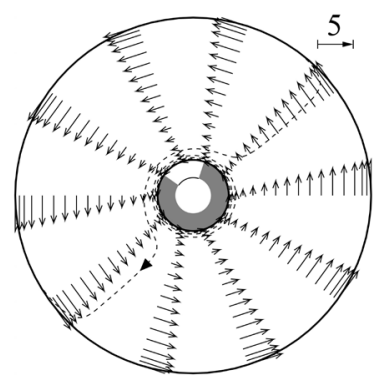

(d) $K n=0.1, R=5$

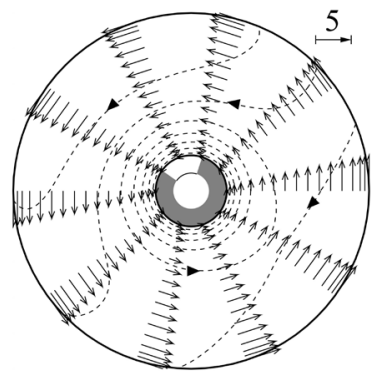

(e) $K n=1, R=5$

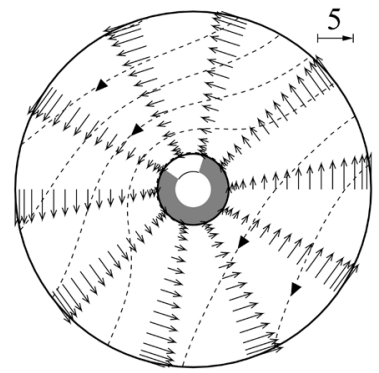

(f) $K n=10, R=5$

Figure 2. Flow fields of Couette flow $\left(\alpha_{1}=0.5, \alpha_{2}=1, C=0.25\right)$. (a, b, c) $R=2$, (d, e, f) $R=5$, (a, d) $K n=0.1$, (b, e) $K n=1$, and (c, f) $K n=10$. Arrow: the normalized flow velocity $\left(u_{1 \mathrm{CF}}, u_{2 \mathrm{CF}}\right) / \Delta_{\mathrm{CF}}$ at its starting point, the magnitude of which is represented by the arrow at the upper right corner of each figure. Dotted line: "stream line", or isoline $\Psi=$ const (Equation (42)), of the heat flow vector; the arrow on the dotted line represents the direction of the heat flow vector. The white part along the surface of the inner cylinder represents the interval of $0.5 \leq \alpha \leq 0.55$.

the results for $R=2$, focusing our attention on the heat flow field. The rotation of the outer cylinder causes the motion of the gas and the heat flow simultaneously. When the Knudsen number is very small, according to the asymptotic theory of the Boltzmann equation [5] [7], the heat flow is present mainly in the Knudsen layers; the heat flow is a clockwise circulation flow along the outer cylinder and is a counterclockwise one along the inner cylinder. The heat flow is enhanced on passing the white part of small accommodation coefficient [29]. Then, the direction of the heat flow slightly shifts from the tangential direction due to the conservation on energy. As the Knudsen number increases (Figure 
2(a)), the heat flow spreads into the bulk region, and forms a roll over the white part. This roll of the heat flow is not special to the present cylindrical Couette flow, but is observed also in the plane Couette flow in Ref. [17]. As the Knudsen number increases further (Figure 2(b)), the outer part of the roll is absorbed by the outer cylinder, and an outflow and an inflow of heat across the cylindrical surface occur. When the Knudsen number is sufficiently large (Figure 2(c)), the roll of the heat flow extends and merges at approximately $\theta=7 \pi / 4$, and a one-way flow of heat from one part to the other part of the outer cylinder is established; the direction is approximately $\left(x_{1}, x_{2}\right)=(-1,-1)$. When the radii ratio is large ( $R=5$, Figures $2(\mathrm{~d})$-(f)), the behavior of the gas is qualitatively similar. For a small Knudsen number (Figure 2(d)), the heat flow is almost absent near the outer cylinder. This is because the breaking effect on the gas motion by the inner cylinder is small, and thus the gas flow near the outer cylinder is nearly a rigid-body rotation; therefore, the velocity distribution function is nearly Maxwellian, and thus the heat flow vanishes.

From the flow fields in Figure 2, one expects nonuniform distributions of the tangential stress and the heat flux along the cylindrical surfaces, which are examined in Figure 3 in detail. The dimensionless tangential stress $P_{\theta r \mathrm{CF}}$ along the cylindrical surfaces at $\hat{r}=1$ and $\hat{r}=R$ for the case of Figure 2(b) $(K n=1, R=2)$ is shown in Figure 3(a) as a function of the azimuthal angle $\theta$. In this figure, $\hat{r}^{2} P_{\theta r \mathrm{CF}}$ is plotted because $P_{\theta r}$ decays approximately $P_{\theta r} \sim \hat{r}^{-2}$ due to the conservation law (23). Note that $-P_{\theta r \mathrm{CF}}>0$ means that the tangential stress acts in the $+\theta(-\theta)$ direction on the inner (outer) cylinder. The magnitude of the tangential stress on the inner cylinder is minimum at approximately $\theta=\pi / 2$, i.e., the minimum position of $\alpha(\theta)$. The dotted line represents the average $-\left(2 \pi \Delta_{\mathrm{CF}}\right)^{-1} \int_{0}^{2 \pi} \hat{r}^{2} P_{\theta r \mathrm{CF}} \mathrm{d} \theta=(2 \pi)^{-1} \mathcal{T}_{\mathrm{CF}}$, where $\mathcal{T}_{\mathrm{CF}}$ is the normalized torque defined by (25). The dotted lines at $\hat{r}=1$ and $\hat{r}=R$ are indistinguishable according to the conservation law (23). Incidentally from Figure 3(a), we can estimate that the inner cylinder is subject to a force (24) from the gas as follows. The tangential stress in the hemicircle of approximately $\pi / 4<\theta<5 \pi / 4$ is less than the average. Therefore, the contribution to the force from this hemicircle and that from the other do not cancel out. Consequently, a force approximately in the direction of $\left(x_{1}, x_{2}\right)=(1,1)$, or $\theta=\pi / 4$, is expected. The force acting on the inner cylinder is discussed in detail in Section 4.6.

Similarly, the normal component $Q_{r \mathrm{CF}}$ of the dimensionless heat flow vector on the cylinders for the same case (Figure 2(b)) is presented in Figure 3(b). Note that $\hat{r} Q_{r \mathrm{CF}}$ is plotted (Equation (23)), and that $Q_{r \mathrm{CF}}>0$ means a heat flow from the cylinder to the gas (from the gas to the cylinder) on the inner (outer) cylinder. Obviously, there is a distribution of inflow and outflow of heat along the cylindrical surfaces. The heat transfer is significant on the outer cylinder, which is easily seen from the flow field in Figure 2(b). The dotted line represents the average $\left(2 \pi \Delta_{\mathrm{CF}}\right)^{-1} \int_{0}^{2 \pi} \hat{r} Q_{\mathrm{rCF}} \mathrm{d} \theta=-(2 \pi)^{-1} \mathcal{Q}_{\mathrm{CF}}$, where $\mathcal{Q}_{\mathrm{CF}}$ is the 


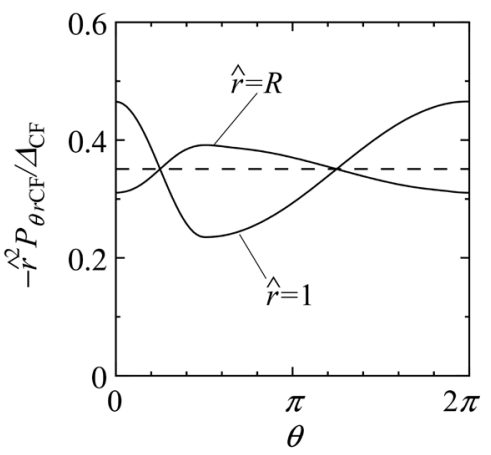

(a)

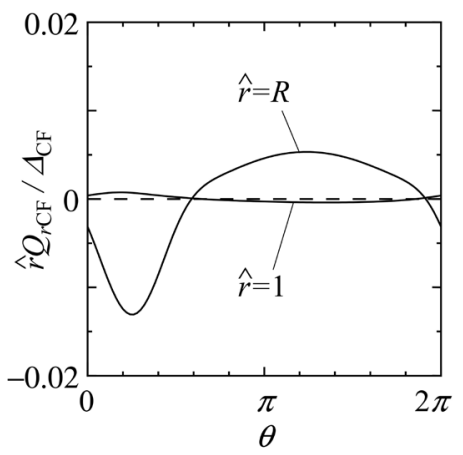

(b)

Figure 3. Distribution of the dimensionless tangential stress $P_{\theta_{r} \mathrm{CF}}$ and the normal component $Q_{r \mathrm{CF}}$ of the heat flow along the cylindrical surfaces in Couette flow ( $K n=1, R=2, \alpha_{1}=0.5, \alpha_{2}=1, C=0.25$; cf. Figure $2(\mathrm{~b})$ ). Solid line in (a): $\hat{r}^{2} P_{\theta r \mathrm{CF}} / \Delta_{\mathrm{CF}}$; solid line in (b): $\hat{r} Q_{r \mathrm{CF}} / \Delta_{\mathrm{CF}}$. Dotted line in (a): the average $-\left(2 \pi \Delta_{\mathrm{CF}}\right)^{-1} \int_{0}^{2 \pi} \hat{r}^{2} P_{\theta r \mathrm{CF}} \mathrm{d} \theta$ at $\hat{r}=1$ and $\hat{r}=R$; dotted line in (b): the average $\left(2 \pi \Delta_{\mathrm{CF}}\right)^{-1} \int_{0}^{2 \pi} \hat{r} Q_{r \mathrm{CF}} \mathrm{d} \theta$ at $\hat{r}=1$ and $\hat{r}=R$; the dotted lines at $\hat{r}=1$ and $\hat{r}=R$ are indistinguishable (Equation (23)).

normalized heat flow rate defined by (26). The dotted lines at $\hat{r}=1$ and $\hat{r}=R$ are indistinguishable according to the conservation law (23). Note that the average, or $\mathcal{Q}_{\mathrm{CF}}$, is very small; to be specific, $(2 \pi)^{-1} \mathcal{Q}_{\mathrm{CF}}$ at $\hat{r}=1$ and $\hat{r}=R$ is less than $0.004 \%$ of the maximum of the integrand $\left|\hat{r} Q_{r \mathrm{CF}} / \Delta_{\mathrm{CF}}\right|\left(=1.3 \times 10^{-2}\right)$ over the cylindrical surfaces. This property is common to every set of the values of the parameters (19); the average $(2 \pi)^{-1} \mathcal{Q}_{\mathrm{CF}}$ is less than $0.15 \%$ of the maximum of $\left|\hat{r} Q_{r \mathrm{CF}} / \Delta_{\mathrm{CF}}\right|$ over all the cases of the parameters (19) conducted in this paper. In view of the accuracy tests in Section 3.3, these small values are of the same order as the inevitable numerical error. Therefore, we may conclude that

$$
\mathcal{Q}_{\mathrm{CF}}=0
$$

irrespective of the values of the parameters (19). That is, although a local heat transfer between the gas and the cylindrical surfaces occurs due to the effect of nonuniform accommodation coefficient, the total heat transfer between the two cylinders vanishes identically. Note that, nevertheless, a one-way heat flow from one part to the other part of the outer cylinder is present, as shown in Figure 2 and Figure 3(b).

\subsection{Heat Transfer Problem}

Next, some examples of the flow field of the heat transfer problem are presented in Figure 4. The parameters (19) in Figures 4(a)-(f) are the same as those in Figures 2(a)-(f). In the figures, the isothermal line $\tau_{\mathrm{HT}}=$ const is represented by the solid line. The meanings of other lines and symbols are similar to those in Figure 2. Due to an effect of the nonuniform accommodation coefficient, a flow is induced between the cylinders at rest kept at different temperatures. The mechanism of this flow is similar to that in the plane heat transfer problem [17]. 


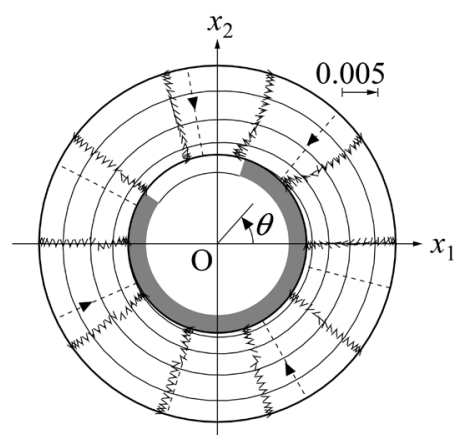

(a) $K n=0.1, R=2$

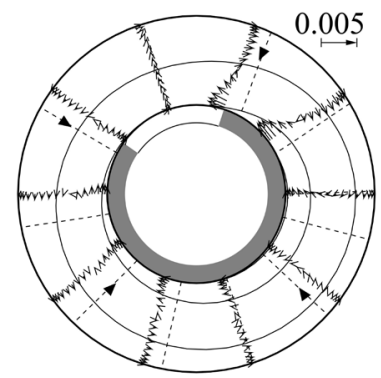

(b) $K n=1, R=2$

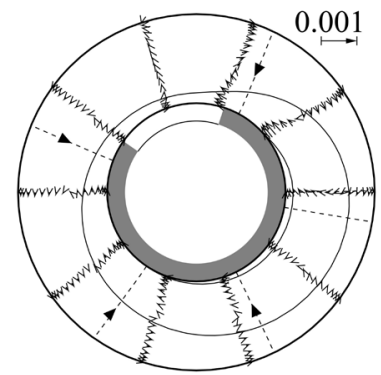

(c) $K n=10, R=2$

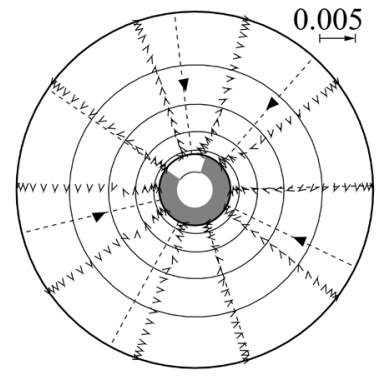

(d) $K n=0.1, R=5$

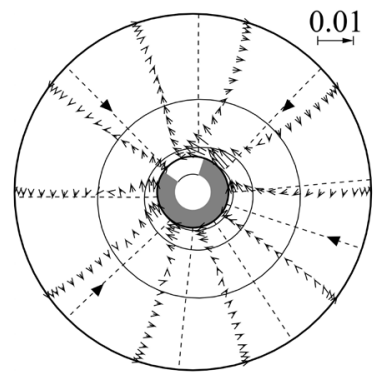

(e) $K n=1, R=5$

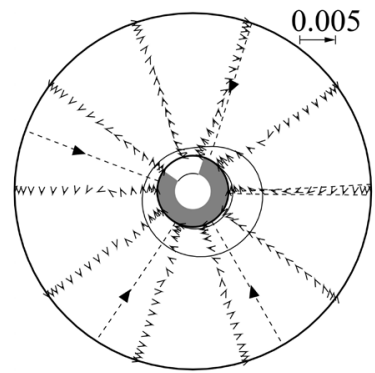

(f) $K n=10, R=5$

Figure 4. Flow fields of the heat transfer problem $\left(\alpha_{1}=0.5, \alpha_{2}=1, C=0.25\right)$. Arrow: the normalized flow velocity $\left(u_{1 \mathrm{HT}}, u_{2 \mathrm{HT}}\right) / \Delta_{\mathrm{HT}}$; dotted line: "stream line" of the heat flow vector $\left(Q_{1 \mathrm{HT}}, Q_{2 \mathrm{HT}}\right)$; solid line: isothermal lines $\tau_{\mathrm{HT}}=0.8,0.6, \cdots$ from the outer. See the caption of Figure 2.

Because of the nonuniformness of the accommodation coefficient, the temperature jump of the gas at the cylindrical surfaces is nonuniform. Consequently, a temperature distribution of the gas is formed along the cylindrical surfaces; the temperature of the gas on the inner cylinder is the highest near the white part of small accommodation coefficient. Then, gas flows are induced along the cylindrical surfaces toward the hot part by the same mechanism as that of the thermal creep flow. Two major flows from both sides along the inner cylinder collide at approximately $\theta=3 \pi / 4$, and finally two rolls are formed in the annulus; the one in approximately $3 \pi / 4<\theta<7 \pi / 4$ is counterclockwise and the other is clockwise. Incidentally, from Figure 4, it is expected that the inner cylinder is subject to a force approximately in the direction of $\left(x_{1}, x_{2}\right)=(1,-1)$ by the 
reaction of these thermal-creep type flows. The force on the inner cylinder will be presented in Section 4.6.

The distributions of the tangential stress $P_{\theta r \mathrm{HT}}$ and the normal component $Q_{r \mathrm{HT}}$ of the heat flow vector along the cylindrical surfaces for the case of Figure 4(b) are presented in Figure 5(a) and Figure 5(b), respectively. The dotted lines represent the averages $\left(2 \pi \Delta_{\mathrm{HT}}\right)^{-1} \int_{0}^{2 \pi} \hat{r}^{2} P_{\theta r \mathrm{HT}} \mathrm{d} \theta=-(2 \pi)^{-1} \mathcal{T}_{\mathrm{HT}}$ and $-\left(2 \pi \Delta_{\mathrm{HT}}\right)^{-1} \int_{0}^{2 \pi} \hat{r} Q_{r \mathrm{HT}} \mathrm{d} \theta=(2 \pi)^{-1} \mathcal{Q}_{\mathrm{HT}}$, respectively (see (25) and (26)). The average value of the tangential stress, or the normalized torque $\mathcal{T}_{\mathrm{HT}}$, is very small, and this property is common to every set of the values of the parameters (19). To be specific, the value $(2 \pi)^{-1} \mathcal{T}_{\mathrm{HT}}$ is less than $0.03 \%$ of the maximum of the integrand $\left|\hat{r}^{2} P_{\theta r \mathrm{HT}}\right|$ over the cylindrical surfaces for every set of the values of the parameters (19). Therefore, following the same criterion as that given in the discussion on Figure 3(b), we concluded that

$$
\mathcal{T}_{\text {HT }}=0
$$

irrespective of the values of the parameters (19). That is, although a local tangential stress is induced on the cylinders, the total torque acting on the inner cylinder vanishes identically irrespective of the parameters (19). Equation (44) is a counterpart of (43).

\subsection{Thermal Creep Flow}

Similarly, some examples of the flow field of the thermal creep flow are presented in Figure 6. Note that the distributions of the dimensionless wall temperature $\tau_{w 0}$ and $\tau_{w 1}$ are $\tau_{w 0}=\tau_{w 1}=\Delta_{\mathrm{TC}} x_{1}$ in the Cartesian coordinates. Thermal creep flows are induced by this temperature distribution along the four parts of the cylindrical surfaces: the $x_{2}>0$ parts of the surfaces of the outer and inner cylinders, and the $x_{2}<0$ parts of those of the outer and inner cylinders. These flows interact, and a flow field of multi rolls is formed. Note

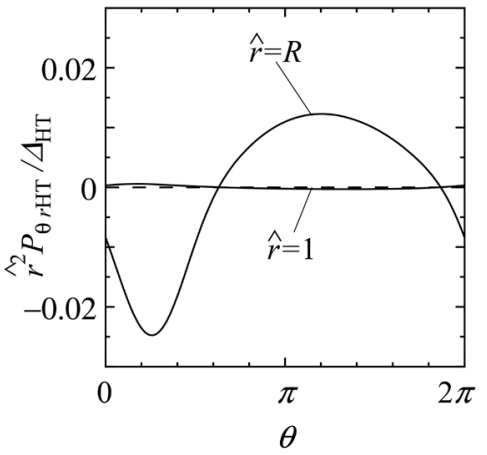

(a)

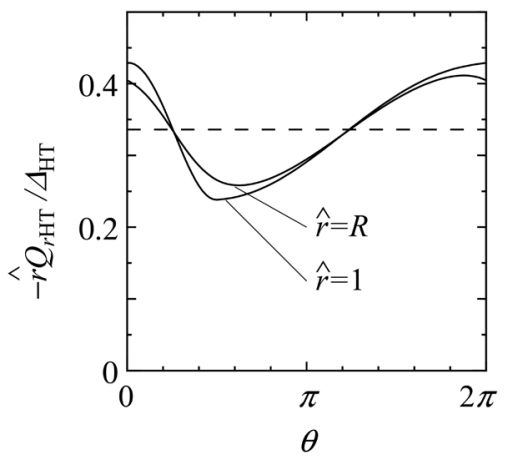

(b)

Figure 5. Distribution of the dimensionless tangential stress $P_{\theta r \mathrm{HT}}$ and the normal component $Q_{r \mathrm{HT}}$ of the heat flow along the cylindrical surfaces in the heat transfer problem ( $K n=1, R=2, \alpha_{1}=0.5, \alpha_{2}=1, C=0.25$; cf. Figure $4(\mathrm{~b})$ ). Solid line in (a): $\hat{r}^{2} P_{\theta r \mathrm{HT}} / \Delta_{\mathrm{HT}}$; solid line in (b): $\hat{r} Q_{r \mathrm{HT}} / \Delta_{\mathrm{HT}}$. Dotted line: the average at $\hat{r}=1$ and $\hat{r}=R$. See the caption of Figure 3 . 


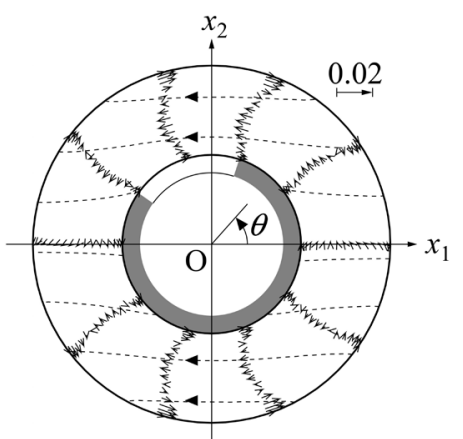

(a) $K n=0.1, R=2$

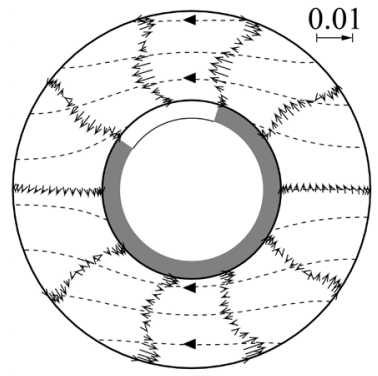

(b) $K n=1, R=2$

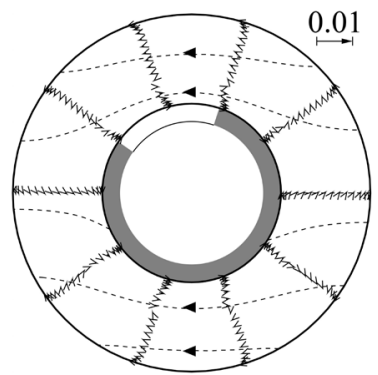

(c) $K n=10, R=2$

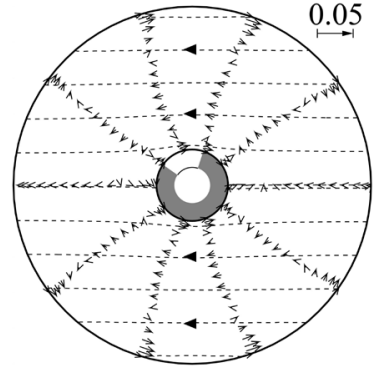

(d) $K n=0.1, R=5$

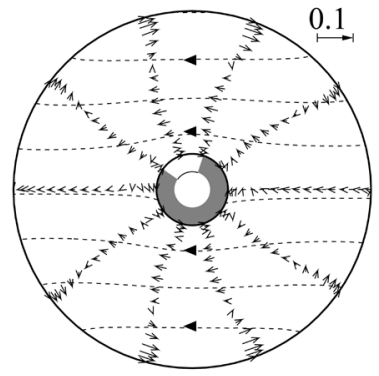

(e) $K n=1, R=5$

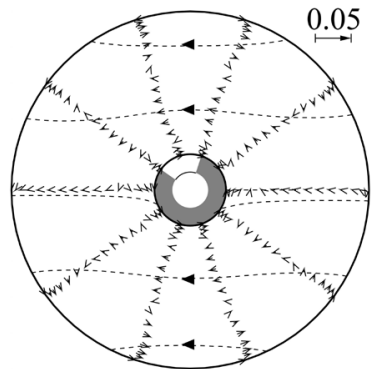

(f) $K n=10, R=5$

Figure 6. Flow fields of the thermal creep flow $\left(\alpha_{1}=0.5, \alpha_{2}=1, C=0.25\right)$. Arrow: the normalized flow velocity $\left(u_{1 \mathrm{TC}}, u_{2 \mathrm{TC}}\right) / \Delta_{\mathrm{TC}}$; dotted line: "stream line" of the heat flow vector $\left(Q_{1 \mathrm{TC}}, Q_{2 \mathrm{TC}}\right)$. See the caption of Figure 2 .

that these thermal creep flows are induced even in the conventional case. The effect of the nonuniform accommodation coefficient works to make the flow asymmetric with respect to $x_{2}=0$. The degree of asymmetry is, however, not so strong. The heat flow, denoted by the dotted line, is basically a one-way flow from the hotter side to the colder side of the outer cylinder.

The distributions of the tangential stress $P_{\theta r \mathrm{TC}}$ and the normal component $Q_{r \mathrm{TC}}$ of the heat flow vector along the cylindrical surfaces for the case of Figure 6(b) are presented in Figure 7(a) and Figure 7(b), respectively. Note that $P_{\theta r \mathrm{TC}} / \sin \theta$ and $Q_{r \mathrm{TC}} / \cos \theta$ are independent of $\theta$ in the conventional case (cf. Section 2.4). A weak deviation from this $\theta$-dependence is observed in Figure 7 (a) and Figure $7(\mathrm{~b})$. The dotted lines represent the averages $\left(2 \pi \Delta_{\mathrm{TC}}\right)^{-1} \int_{0}^{2 \pi} \hat{r}^{2} P_{\theta r \mathrm{TC}} \mathrm{d} \theta=-(2 \pi)^{-1} \mathcal{T}_{\mathrm{TC}}$ and $\left(2 \pi \Delta_{\mathrm{TC}}\right)^{-1} \int_{0}^{2 \pi} \hat{r} Q_{r \mathrm{TC}} \mathrm{d} \theta=-(2 \pi)^{-1} \mathcal{Q}_{\mathrm{TC}}$, 
respectively (see (25) and (26)). Note that the average values are small but do not vanish. To be specific, $-(2 \pi)^{-1} \mathcal{T}_{\text {TC }}=5.394 \times 10^{-3}$ at $\hat{r}=1$ and $5.392 \times 10^{-3}$ at $\hat{r}=R \quad$ in Figure $7(\mathrm{a})$, and $-(2 \pi)^{-1} \mathcal{Q}_{\mathrm{TC}}=-2.112 \times 10^{-2}$ at $\hat{r}=1$ and $-2.105 \times 10^{-2}$ at $\hat{r}=R$ in Figure $7(\mathrm{~b})$. The magnitudes of these values are much larger than the estimated error (cf. Section 3.3). The values at $\hat{r}=1$ and $\hat{r}=R$ agree well; the relative differences are only $0.04 \%$ and $0.3 \%$, respectively, which are of the same order as the numerical error. We also obtain nonzero values of $\mathcal{T}_{\mathrm{TC}}$ and $\mathcal{Q}_{\mathrm{TC}}$ for every set of the values of the parameters (19) except $\mathcal{T}_{\text {TC }}$ at $C=0.5$ (Equation (37)). That is, we find that both the torque $\mathcal{T}_{\text {TC }}$ and the heat flow rate $\mathcal{Q}_{\mathrm{TC}}$ in the thermal creep flow do not generally vanish in contrast to (43) and (44). These two quantities appear in the reciprocity relations (28) and (29). In this way, we obtain two nondegenerate reciprocity relations by the effect of nonuniform accommodation coefficient. The reciprocity relations (28) and (29) are numerically confirmed in the next subsection. From Figure 7(a), incidentally, we can estimate the force (24) acting on the inner cylinder as follows. The contribution of the tangential stress from $0<\theta<\pi$ and that from $\pi<\theta<2 \pi$ combine to produce a resultant force approximately in the $-x_{1}$ direction. Note that, in contrast to Couette flow and heat transfer problems, a force acts even in the conventional thermal creep flow; this force is similar to the thermal force on a body with a nonuniform surface temperature, e.g., in Ref. [7]. From Figure 7(a), it is expected that the direction of the force is weakly affected by the nonuniformness of the accommodation coefficient.

\subsection{Reciprocity Relations}

The reciprocity relations (27), (28), and (29) are numerically confirmed now. From (43) and (44), which are numerically obtained in Sections 4.1 and 4.2, we directly have

$$
\mathcal{Q}_{\mathrm{CF}}(R)=-\mathcal{T}_{\mathrm{HT}}(R)=0
$$

irrespective of the parameters (19). We now examine the other two relations (28) and (29), which do not generally vanish, over the range of the parameters stated at the beginning of Section 4 except $C=0.5$. From the numerical results, the relative difference between the left-hand and right-hand sides of (28) is less than $1.0 \%$; the relative difference between the left-hand and right-hand sides of (29) is less than $0.43 \%$. In view of the accuracy tests in Section 3.3, these small differences are of the order of the numerical error. Therefore, we may conclude that the reciprocity relations (28) and (29) are numerically confirmed over the above-mentioned range of the parameters (19). It may be noted that the relative agreement in the nonvanishing equalities (28) and (29) is worse than the corresponding reciprocity equalities in the plane channel flows [17]. The reason may be attributed to the difficulty in obtaining accurate numerical solutions at small Knudsen numbers and a large radii ratio in the present paper (see the last paragraph in Section 3.3). 


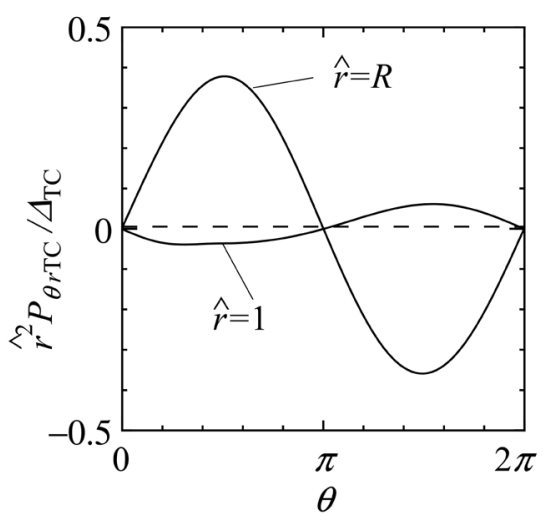

(a)

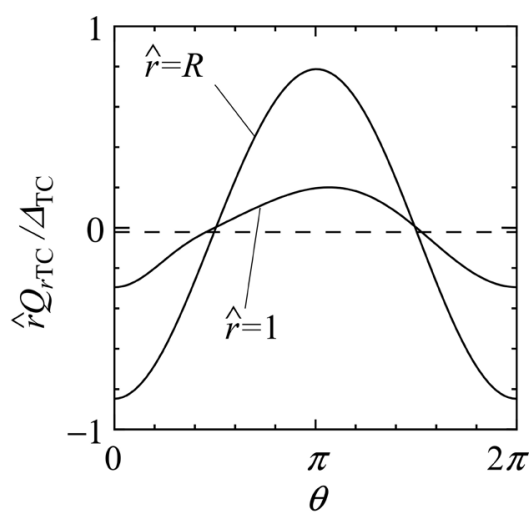

(b)

Figure 7. Distribution of the dimensionless tangential stress $P_{\theta r \mathrm{TC}}$ and the normal component $Q_{r \mathrm{TC}}$ of the heat flow along the cylindrical surfaces in the thermal creep flow ( $K n=1, R=2, \alpha_{1}=0.5, \alpha_{2}=1, C=0.25$; cf. Figure $6(\mathrm{~b})$ ). Solid line in (a): $\hat{r}^{2} P_{\theta r \mathrm{TC}} / \Delta_{\mathrm{TC}}$; solid line in (b): $\hat{r} Q_{r \mathrm{TC}} / \Delta_{\mathrm{TC}}$. Dotted line: the average at $\hat{r}=1$ and $\hat{r}=R$. See the caption of Figure 3.

When the accommodation coefficient $\alpha(\theta)$ is uniform or symmetric with respect to $\pi$, both sides of all the reciprocity relations (27), (28), and (29) vanish identically (Section 2.4). In this paper, therefore, we especially considered the nonuniform and asymmetric distribution of the accommodation coefficient to avoid the degeneracy of the reciprocity relations. As a result, we obtained nondegenerate relations (28) and (29), although the magnitude is small. Nevertheless, both sides of (27) vanish irrespective of the values of the parameters (19). Note that the meaning of the second equality in (45) is completely different from that in (32), (34), (36), and (37) in the conventional or symmetric case; the integrands $Q_{r \mathrm{CF}}$ and $P_{\theta r \mathrm{HT}}$ of $\mathcal{Q}_{\mathrm{CF}}$ and $\mathcal{T}_{\mathrm{HT}}$ do not vanish identically nor do not have any antisymmetry with respect to $\theta$ (cf. Figure 3(b) and Figure 5(a)). In the plane channel flow problem in Ref. [17], a degeneracy corresponding to (45) was found between plane Couette flow and plane heat transfer problems. To clarify whether a similar property holds also in the flows between coaxial cylinders or not is one of the important goals of the present paper. The answer turned out to be affirmative. This result suggests that this degeneracy property may have a universality over a wider class of flows beyond the flows in a plane channel or an annulus.

In rarefied gas dynamics, a phenomenon referred to as "nonexistence of one-way flow" is known [7]. Consider a rarefied gas in a straight pipe with an arbitrary cross section, an arbitrary uniform accommodation coefficient, and an arbitrary periodic surface temperature distribution in the longitudinal direction. Then, a flow is induced due to a rarefaction effect. However, net mass flow is never induced through the pipe. The degeneracy properties observed in the present paper show a similarity to this phenomenon, and there may be some relation between them [17]. 


\subsection{Torque on the Inner Cylinder and the Heat Flow Rate between the Cylinders}

The torque acting on the inner cylinder and the heat flow rate between the two cylinders in the three flow problems are presented here over a wide range of the parameters. In the rest of Section 4, the Knudsen number

$$
K n_{D}=\frac{\ell}{r_{1}-r_{0}}=\frac{K n}{R-1}
$$

based on the gap size $r_{1}-r_{0}$ is used to present the results, because it is convenient to observe the similarity between the results for different radii ratios $R$. The normalized torque $\mathcal{T}_{\text {CF }}$ of Couette flow is presented in Figure 8 as a function of $K n_{D}$ for various values of $R$ and $C$. Note that the parameter $C$ represents the degree of asymmetry of the accommodation coefficient $\alpha(\theta)$ (see the explanation below (19)). The markers represent the numerical results. The torque is an increasing function of both the Knudsen number $K n_{D}$ and the radii ratio $R$. The torque is, however, almost independent of the parameter $C$; a similar property was observed also in plane Couette flow [17]. The solid line represents the estimate formula proposed in Ref. [14]:

$$
\mathcal{T}_{\mathrm{CF}}=\eta \mathcal{T}_{\mathrm{CF} 1}+(1-\eta) \mathcal{T}_{\mathrm{CF} 2},
$$

where $\mathcal{T}_{\mathrm{CF} 1}$ and $\mathcal{T}_{\mathrm{CF} 2}$ are normalized torque in the conventional Couette flow problem with $\alpha(\theta)=$ const $=\alpha_{1}$ and $\alpha(\theta)=$ const $=\alpha_{2}$, respectively. The factor $\eta$ is the constant defined by

$$
\eta \alpha_{1}+(1-\eta) \alpha_{2}=\frac{1}{2 \pi} \int_{0}^{2 \pi} \alpha(\theta) \mathrm{d} \theta .
$$

For (2), $\eta=1 / 2$ irrespective of $C$. The estimate formula approximates the numerical results very well.

Next, the normalized heat flow rate $\mathcal{Q}_{\mathrm{HT}}$ between the cylinders (from the outer cylinder to the inner) in the heat transfer problem is presented in Figure 9. The heat flow rate is an increasing function of both $K n_{D}$ and $R$. A very weak dependence on the parameter $C$ is observed also in this heat flow rate. The solid line represents the estimate formula, i.e., the counterpart of (47). The estimate formula approximates the numerical results very well.

Similarly, the normalized torque $\mathcal{T}_{\text {TC }}$ and the heat flow rate $\mathcal{Q}_{\mathrm{TC}}$ in the thermal creep flow are presented in Figure 10(a) and Figure 10(b), respectively. The torque $\mathcal{T}_{\text {TC }}$ and the heat flow rate $\mathcal{Q}_{\mathrm{TC}}$ vanish in the conventional case (Equation (35)). In other words, the nonzero values in Figure 10(a) and Figure 10 (b) are essentially due to the effect of nonuniform accommodation coefficient. (Note that the dotted lines in Figure 7(a) and Figure 7(b) are divided by $2 \pi$.) The torque and the heat flow rate increase with an increase in the Knudsen number. The dependence on the parameter $C$ is perceptible. When $C$ increases to approach 0.5 , the torque $-\mathcal{T}_{\mathrm{TC}}$ decreases to vanish (Equation (37)), whereas the heat flow rate $\mathcal{Q}_{\mathrm{TC}}$ increases and takes the maximum when $C=0.5$. The estimate formula, the counterpart of (47), is omitted because it vanishes identi- 
cally.

\subsection{Force Acting on the Inner Cylinder}

Finally, the force acting on the inner cylinder in the three flow problems is presented in Figure 11. The figure shows the dimensionless magnitude $\left|\hat{F}_{\mathrm{J}}\right|$ and the direction angle $\vartheta_{\mathrm{J}} \quad(\mathrm{J}=\mathrm{CF}, \mathrm{HT}$, and TC) of the force defined by

$$
F_{1 \mathrm{~J}} /\left(p_{0} r_{0}\right)=\left|\hat{F}_{\mathrm{J}}\right| \cos \vartheta_{\mathrm{J}}, \quad F_{2 \mathrm{~J}} /\left(p_{0} r_{0}\right)=\left|\hat{F}_{\mathrm{J}}\right| \sin \vartheta_{\mathrm{J}}, \quad-0.9 \pi<\vartheta_{\mathrm{J}} \leq 1.1 \pi
$$

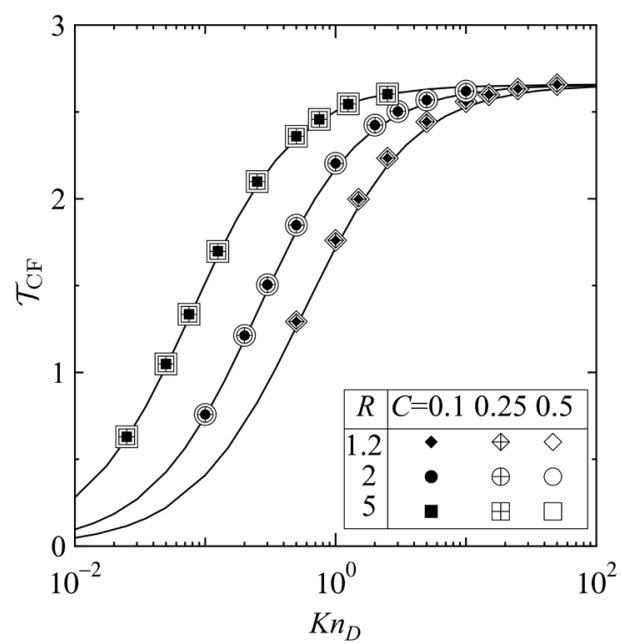

Figure 8. Normalized torque $\mathcal{T}_{\mathrm{CF}}$ acting on the inner cylinder as a function of $K n_{D}, R$, and $C$ in Couette flow $\left(\alpha_{1}=0.5, \alpha_{2}=1\right)$. Markers: numerical solutions; diamonds for $R=1.2$, circles for $R=2$, and squares for $R=5$; closed symbols for $C=0.1$, symbols with plus for $C=0.25$, and open symbols for $C=0.5$ (see the key in the figure). Solid line: estimate formula (47) for $R=1.2,2$, and 5 from the bottom.

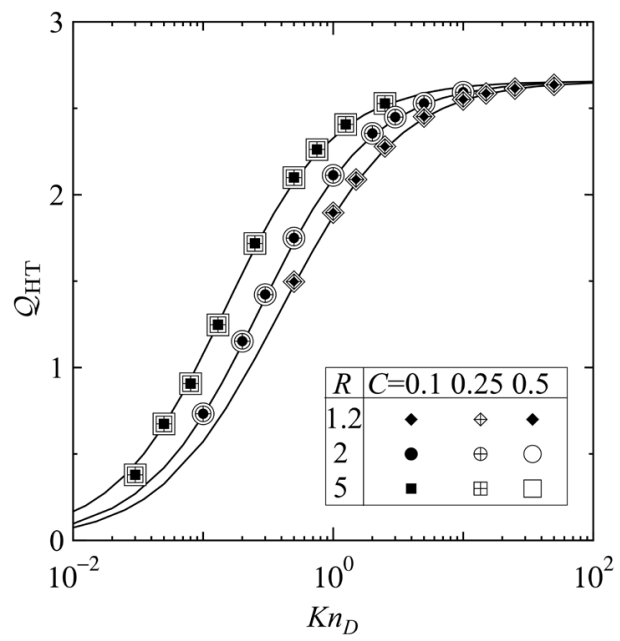

Figure 9. Normalized heat flow rate $\mathcal{Q}_{\mathrm{HT}}$ between the cylinders as a function of $K n_{D}, R$, and $C$ in the heat transfer problem $\left(\alpha_{1}=0.5, \alpha_{2}=1\right)$. Markers: numerical solutions (see the key in the figure). Solid line: estimate formula (cf. (47)) for $R=1.2,2$, and 5 from the bottom. 


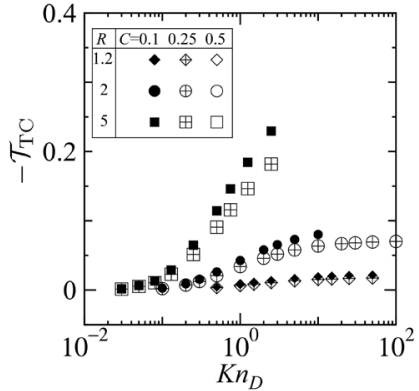

(a)

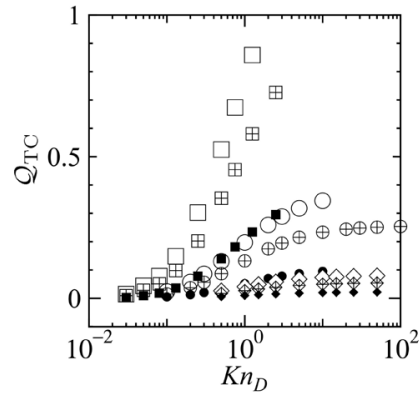

(b)

Figure 10. Normalized torque $\mathcal{T}_{\text {TC }}$ acting on the inner cylinder and the heat flow rate $\mathcal{Q}_{\mathrm{TC}}$ between the cylinders as a function of $K n_{D}, R$, and $C$ in the thermal creep flow $\left(\alpha_{1}=0.5, \alpha_{2}=1\right)$. (a) Normalized torque $\mathcal{T}_{\mathrm{TC}}$ and (b) the heat flow rate $\mathcal{Q}_{\mathrm{TC}}$. Markers: numerical solution (see the key in the figure). The $\mathcal{T}_{\text {TC }}$ vanishes when $C=0.5$ (Equation (37)).
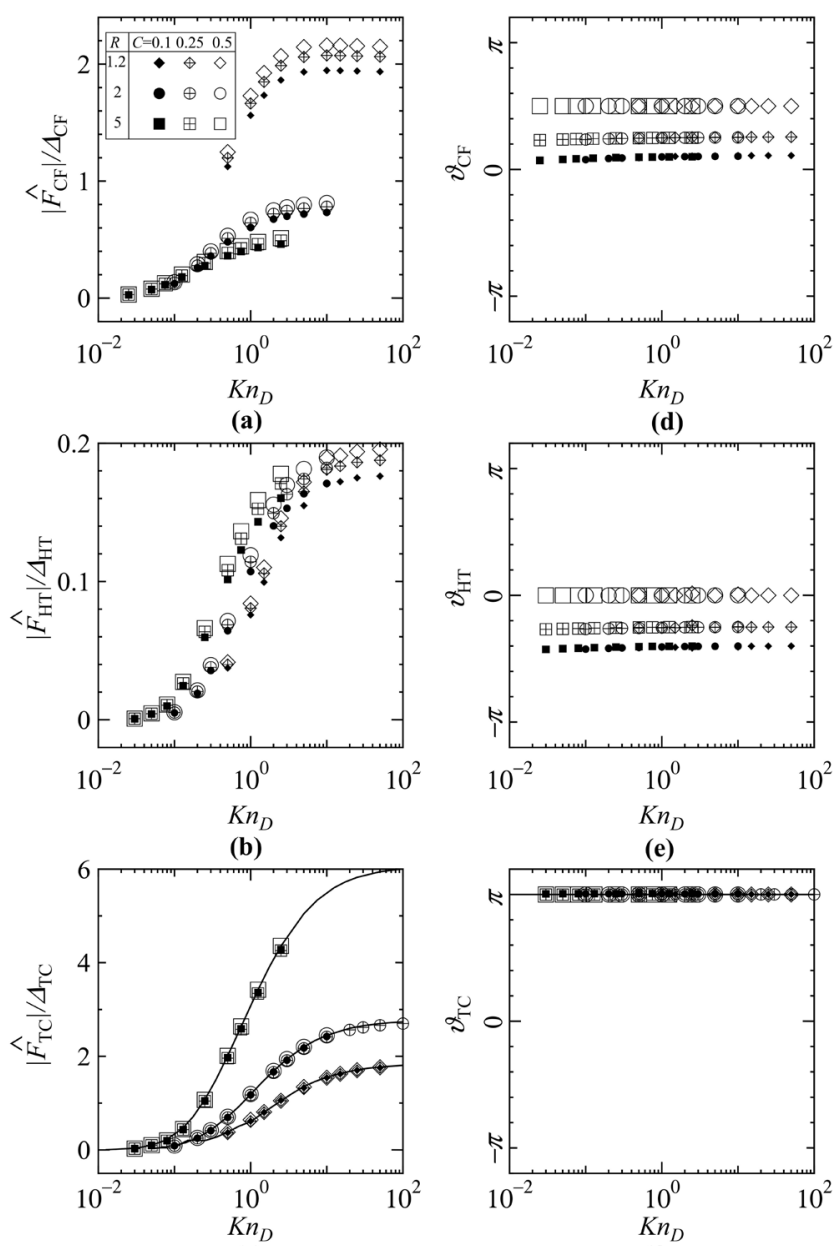

(e)

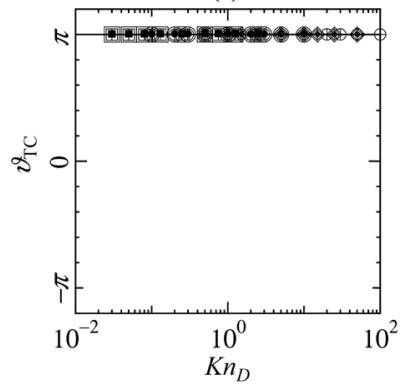

(c)

(f)

Figure 11. Dimensionless force acting on the inner cylinder as a function of $K n_{D}, R$, and $C\left(\alpha_{1}=0.5, \alpha_{2}=1\right)$. (a, b, c) The magnitude $\left|\hat{F}_{\mathrm{J}}\right|$ and $(\mathrm{d}, \mathrm{e}, \mathrm{f})$ the direction $\vartheta_{\mathrm{J}}$ (Equation (49)). (a, d) Couette flow, (b, e) heat transfer problem, and (c, f) thermal creep flow. Markers: numerical solution (see the key in Figure 11(a)). Solid line in Figure 11(c) and Figure 11(f): estimate formula (cf. (47)) for $R=1.2,2$, and 5 from the bottom. 
as functions of the Knudsen number $K n_{D}$. In the conventional Couette flow and the heat transfer problems, the flow is axially symmetric and, obviously, the inner cylinder is subject to no force. That is, the forces in these two problems, which are shown in Figures 11(a)-(e), arise essentially due to the effect of nonuniform accommodation coefficient. The magnitude $\left|\hat{F}_{\mathrm{J}}\right|$ is an increasing function of the Knudsen number $K n_{D}$; the dependence on the parameter $C$ is perceptible (Figure 11(a) and Figure 11(b)). In contrast, the direction $\vartheta_{\mathrm{J}}$ is only weakly dependent on $K n_{D}$ and $R$, and is determined almost solely by $C$. In Couette flow at $C=0.25$ (Figure 11(d)), the direction is approximately $\vartheta_{\mathrm{CF}}=\pi / 4$, which agrees with the estimate given in the discussion on Figure 3(a). As $C$ increases and approaches 0.5 , the position $\theta$ of the minimum of the accommodation coefficient approaches $\pi$, and thus the direction $\vartheta_{\mathrm{CF}}$ of the force increases and approaches $\vartheta_{\mathrm{CF}}=\pi / 2$ (Equation (36)). The direction $\vartheta_{\mathrm{HT}}$ in the heat transfer problem increases to approach zero from a negative value as $C$ increases and approaches 0.5 (Equation (37)). This behavior may also be understood from Figure 4.

In the thermal creep flow in Figure 11(c) and Figure 11(f), in contrast, the cylinder is subject to a force even in the conventional case. The influence of the nonuniform accommodation coefficient on the direction $\vartheta_{\mathrm{TC}}$ of the force is weak (Figure 11(f)), in agreement with the estimate in the discussion on Figure 7 (a). The direction $\vartheta_{\text {TC }}$ is nearly equal to $\pi$, which is the direction in the conventional case (Equation (35)). The solid line represents the estimate formula corresponding to (47). The estimate formula approximates the numerical results very well.

Couette flow considered in this paper is a simple model of micro lubrication between a journal and a bearing in which the journal is partially soiled or fabricated with various materials. As shown in Figure 11, a force acting on the inner cylinder arises due to the effect of nonuniform surface properties of the journal. Note that the magnitude of the force is not weak. For example, the magnitude of the force is approximately $95 \%$ of the torque divided by the radius of the inner cylinder when $K n_{D}=1, R=1.2$, and $C=0.25$; the magnitude of the force increases as the clearance between the cylinders shrinks (or $R$ decreases). This phenomenon poses a serious problem in micro engineering, because this force may cause an eccentricity of the journal and may affect the lubrication process. In this study, however, the model is not sufficiently good for some reasons, e.g., the inner cylinder is at rest, and the gap is not so narrow. A more realistic study focusing on the engineering applications, which is beyond the scope of this paper, will be conducted in a separate paper.

\section{Conclusion}

In this paper, we studied the flows of a rarefied gas between coaxial circular cylinders with nonuniform surface properties on the basis of kinetic theory. We assumed that the outer cylinder is a diffuse reflection boundary and the inner 
cylinder is a Maxwell-type boundary whose accommodation coefficient varies in the circumferential direction. Couette flow, the thermal creep flow, and the heat transfer problem were studied. The linearized ES-BGK model of the Boltzmann equation was numerically analyzed using the finite difference method. The time-independent behavior of the gas was studied over a wide range of the Knudsen number, radii ratio, and the parameter characterizing the distribution of the accommodation coefficient. Due to the effect of nonuniform surface properties, a local heat transfer between the gas and the cylindrical surface occurs in Couette flow; a local tangential stress arises in the heat transfer problem. However, the total heat transfer between the two cylinders in Couette flow and the total torque acting on the inner cylinder in the heat transfer problem vanish irrespective of the flow parameters. Two nondegenerate reciprocity relations arise due to the effect of nonuniform surface properties. The reciprocity relations among the above-mentioned three flows were numerically confirmed over a wide range of the flow parameters. The force acting on the inner cylinder, which also arises due to the effect of nonuniform surface properties in Couette flow and the heat transfer problems, was studied in detail.

\section{Acknowledgements}

This work was supported by JSPS KAKENHI Grant (18K03949).

\section{Conflicts of Interest}

The author declares no conflicts of interest regarding the publication of this paper.

\section{References}

[1] Kogan, M. (1969) Rarefied Gas Dynamics. Plenum Press, New York. https://doi.org/10.1007/978-1-4899-6381-9

[2] Cercignani, C. (1988) The Boltzmann Equation and Its Applications. Springer, New York. https://doi.org/10.1007/978-1-4612-1039-9

[3] Sharipov, F. and Seleznev, V. (1998) Data on Internal Rarefied Gas Flows. Journal of Physical and Chemical Reference Data, 27, 657-706. https://doi.org/10.1063/1.556019

[4] Cercignani, C. (2000) Rarefied Gas Dynamics. Cambridge University Press, Cambridge.

[5] Sone, Y. (2002) Kinetic Theory and Fluid Dynamics. Birkhäuser, New York. https://doi.org/10.1007/978-1-4612-0061-1

[6] Karniadakis, G., Beskok, A. and Aluru, N. (2005) Microflows and Nanoflows: Fundamentals and Simulation. Springer, New York.

[7] Sone, Y. (2007) Molecular Gas Dynamics. Birkhäuser, New York. https://doi.org/10.1007/978-0-8176-4573-1

[8] Veijola, T., Kuisma, H. and Lahdenperä, J. (1998) The Influence of Gas-Surface Interaction on Gas-Film Damping in a Silicon Accelerometer. Sensors and Actuators $A, 66,83-92$. https://doi.org/10.1016/S0924-4247(97)01732-9 
[9] Kang, S.C., Crone, R.M. and Jhon, M.S. (1999) A New Molecular Gas Lubrication Theory Suitable for Head-Disk Interface Modeling. Journal of Applied Physics, 85, 5594-5596. https://doi.org/10.1063/1.369810

[10] Cercignani, C., Lampis, M. and Lorenzani, S. (2004) Plane Poiseuille Flow with Symmetric and Nonsymmetric Gas-Wall Interactions. Transport Theory and Statistical Physics, 33, 545-561. https://doi.org/10.1081/TT-200053939

[11] Scherer, C.S., Prolo Filho, J.F. and Barichello, L.B. (2009) An Analytical Approach to the Unified Solution of Kinetic Equations in Rarefied Gas Dynamics. I. Flow problems. Journal of Applied Mathematics and Physics (ZAMP), 60, 70-115. https://doi.org/10.1007/s00033-008-7084-4

[12] Doi, T. (2014) Plane Poiseuille Flow and Thermal Transpiration of a Highly Rarefied Gas between the Two Walls of Maxwell-Type Boundaries with Different Accommodation Coefficients: Effect of a Weak External Force. Journal of Applied Mathematics and Physics (ZAMP), 66, 1805-1820. https://doi.org/10.1007/s00033-014-0454-1

[13] Doi, T. (2016) Transient Couette Flow of a Rarefied Gas between Plane Parallel Walls with Different Surface Properties. Physics of Fluids, 28, Article ID: 022006. https://doi.org/10.1063/1.4941738

[14] Doi, T. (2015) Poiseuille Flow and Thermal Transpiration of a Rarefied Gas between Parallel Plates: Effect of Nonuniform Surface Properties of the Plates in the Transverse Direction. ASME Journal of Fluids Engineering, 137, Article ID: 101103. https://doi.org/10.1115/1.4030490

[15] Doi, T. (2015) Poiseuille Flow and Thermal Transpiration of a Rarefied Gas between Parallel Plates II: Effect of Nonuniform Surface Properties in the Longitudinal Direction. Journal of Applied Mathematics and Physics (ZAMP), 66, 3405-3423. https://doi.org/10.1007/s00033-015-0580-4

[16] Kumar, A., Datta, S. and Kalyanasundaram, D. (2016) Permeability and Effective Slip in Confined Flows Transverse to Wall Slippage Patterns. Physics of Fluids, 28, Article ID: 082002. https://doi.org/10.1063/1.4959184

[17] Doi, T. (2017) Reciprocity Relations in Flows of a Rarefied Gas between Plane Parallel Walls with Nonuniform Surface Properties. Journal of Applied Mathematics and Physics, 68, 66. https://doi.org/10.1007/s00033-017-0811-y

[18] Doi, T. (2018) Poiseuille, Thermal Transpiration and Couette Flows of a Rarefied Gas between Plane Parallel Walls with Nonuniform Surface Properties in the Transverse Direction and Their Reciprocity Relations. Fluid Dynamics Research, 50, Article ID: 025502. https://doi.org/10.1088/1873-7005/aa962d

[19] Sharipov, F. (1994) Onsager-Casimir Reciprocity Relations for Open Gaseous Systems at Arbitrary Rarefaction I. General Theory for Single Gas. Physica A, 203, 437-456. https://doi.org/10.1016/0378-4371(94)90009-4

[20] Takata, S. (2009) Symmetry of the Linearized Boltzmann Equation and Its Application. Journal of Statistical Physics, 136, 751-784. https://doi.org/10.1007/s10955-009-9793-2

[21] Holway, J.H. (1966) New Statistical Models for Kinetic Theory: Methods of Construction. Physics of Fluids, 9, 1658-1673. https://doi.org/10.1063/1.1761920

[22] Andries, P., Tallec, P.L., Perlat, J.-P. and Perthame, B. (2000) The Gaussian-BGK Model of Boltzmann Equation with Small Prandtl Number. European Journal of Mechanics B/ Fluids, 19, 813-830. https://doi.org/10.1016/S0997-7546(00)01103-1

[23] Takata, S., Hattori, M. and Hasebe, T. (2016) Slip/Jump Coefficients and Knudsen-Layer Corrections for the ES Model in the Generalized Slip-Flow Theory. AIP 
Conference Proceedings, 1786, Article ID: 040004.

https://doi.org/10.1063/1.4967542

[24] Cercignani, C. and Sernagiotto, F. (1967) Cylindrical Couette Flow of a Rarefied Gas. Physics of Fluids, 10, 1200-1024. https://doi.org/10.1063/1.1762263

[25] Aoki, K., Yoshida, H., Nakanishi, T. and Garcia, A.L. (2003) Inverted Velocity Profile in the Cylindrical Couette Flow of a Rarefied Gas. Physical Review E, 68, Article ID: 016302. https://doi.org/10.1103/PhysRevE.68.016302

[26] Bassanini, P., Cercignani, C. and Pagani, C.D. (1968) Influence of the Accommodation Coefficient on the Heat Transfer in a Rarefied Gas. International Journal of Heat and Mass Transfer, 11, 1359-1369.

https://doi.org/10.1016/0017-9310(68)90181-6

[27] Aoki, K., Sone, Y., Nishino, K. and Sugimoto, H. (1991) Numerical Analysis of Unsteady Motion of a Rarefied Gas Caused by Sudden Changes of Wall Temperature with Special Interest in the Propagation of a Discontinuity in the Velocity Distribution Function. In: Beylich, A.E., Ed., Rarefied Gas Dynamics, VCH, Weinheim, 222-231.

[28] Sugimoto, H. and Sone, Y. (1992) Numerical Analysis of Steady Flows of a Gas Evaporating from Its Cylindrical Condensed Phase on the Basis of Kinetic Theory. Physics of Fluids A, 4, 419-440. https://doi.org/10.1063/1.858313

[29] Wakabayashi, M., Ohwada, T. and Golse, F. (1996) Numerical Analysis of the Shear and Thermal Creep Flows of a Rarefied Gas over the Plane Wall of a Maxwell-Type Boundary on the Basis of the Linearized Boltzmann Equation for Hard-Sphere Molecules. European Journal of Mechanics B/ Fluids, 15, 175-201. 